\title{
A SINFONI view of the nuclear activity and circumnuclear star formation in NGC 4303 - II. Spatially resolved stellar populations
}

\author{
Natacha Z. Dametto ${ }^{\oplus},{ }^{1 \star}$ R. Riffel ${ }^{\oplus},{ }^{1}$ L. Colina,${ }^{2}$ R. A. Riffel ${ }^{\oplus},{ }^{3}$ J. Piqueras López, ${ }^{2}$ \\ R. I. Davies, ${ }^{4}$ L. Burtscher, ${ }^{5}$ R. B. Menezes, ${ }^{6}$ S. Arribas,${ }^{2}$ M. G. Pastoriza, ${ }^{1}$ \\ A. Labiano, ${ }^{7}$ T. Storchi-Bergmann, ${ }^{1}$ L. G. Dahmer-Hahn ${ }^{1}$ and D. A. Sales ${ }^{8}$ \\ ${ }^{1}$ Departamento de Astronomia, Instituto de Física, Universidade Federal do Rio Grande do Sul, CP 15051, Porto Alegre, RS 91501-970, Brazil \\ ${ }^{2}$ Centro de Astrobiología (CAB, CSIC-INTA), Carretera de Ajalvir, E-28850 Torrejón de Ardoz, Madrid, Spain \\ ${ }^{3}$ Departamento de Física, Centro de Ciências Naturais e Exatas, Universidade Federal de Santa Maria, Santa Maria, RS 97105900, Brazil \\ ${ }^{4}$ Max Planck Institut für extraterrestrische Physik, Postfach 1312, D-85741, Garching, Germany \\ ${ }^{5}$ Leiden Observatory, Leiden University, PO Box 9513, NL2300, RA Leiden, the Netherlands \\ ${ }^{6}$ Instituto de Astronomia Geofísica e Ciências Atmosféricas, Universidade de São Paulo, Rua do Matão 1226, São Paulo, SP Brazil \\ ${ }^{7}$ Department of Physics, Institute for Astronomy, ETH Zurich, CH-8093 Zurich, Switzerland \\ ${ }^{8}$ Instituto de Matemática, Estatística e Física, Universidade Federal do Rio Grande, Rio Grande 96203-900, Brazil
}

Accepted 2018 October 31. Received 2018 October 23; in original form 2018 June 20

\begin{abstract}
We present a spatially resolved stellar population study of the inner $\sim 200 \mathrm{pc}$ radius of NGC 4303 based on near-infrared integral field spectroscopy with SINFONI/VLT at a spatial resolution of $40-80 \mathrm{pc}$ and using the STARLIGHT code. We found that the distribution of the stellar populations presents a spatial variation, suggesting an age stratification. Three main structures stand out. Two nuclear blobs, one composed by young stars $(t \leq 50 \mathrm{Myr})$ and one with intermediate-age stars ( $50 \mathrm{Myr}<t \leq 2 \mathrm{Gyr}$ ), both shifted from the centre. The third one is an internal intermediate-age spiral arm-like structure, surrounding the blob of young stars. Our results indicate that star formation has occurred through multiple bursts in this source. Furthermore, the youngest stellar populations $(t \lesssim 2 \mathrm{Gyr})$ are distributed along a circumnuclear star-forming ring with $r \sim 250 \mathrm{pc}$. The ring displays star formation rates (SFRs) in the range of $0.002-0.14 \mathrm{M}_{\odot} \mathrm{yr}^{-1}$, favouring the 'pearls-on-a-string' scenario. The old underlying bulge stellar population component $(t>2 \mathrm{Gyr})$ is distributed outside the two blob structures. For the nuclear region (inner $\sim 60 \mathrm{pc}$ radius) we derived an SFR of $0.43 \mathrm{M}_{\odot} \mathrm{yr}^{-1}$ and found no signatures of non-thermal featureless continuum and hot dust emission, supporting the scenario in which an LLAGN/LINER-like source is hidden in the centre of NGC 4303. Thus, our results reveal a rather complex star formation history in NGC 4303, with different stellar population components coexisting with a low efficiency accreting black hole in its centre.
\end{abstract}

Key words: galaxies: active-galaxies: stellar content-infrared: stars.

\section{INTRODUCTION}

The stellar population synthesis technique is a powerful tool to derive the galaxy star formation history (SFH). Disentangling the intrinsic properties of the galaxy, such as mass, age, metallicity, and dust, is a key step for the understanding of a galaxy formation and evolution. For example, there are physical properties of the galaxy bulges that correlate with properties of the active galactic nuclei (AGNs) they harbour, e.g. the $M_{\bullet}-\sigma_{\star}$ relation (Ferrarese \& Merritt 2000; Gebhardt et al. 2000; Kormendy \& Ho 2013), in which the mass of the supermassive black hole $\left(\mathrm{SMBH}, M_{\bullet}\right)$ correlates with the velocity dispersion of the bulge stars $\left(\sigma_{\star}\right)$. The existence of such correlations suggests a possible causal link between the bulge formation and the central black hole or even that the evolution of both might be regulated by a common effect. A strong candidate to explain the relation between these two phenomena is circumnuclear star formation, since they both depend on the inflow of gas towards the innermost regions of the galaxy (e.g. Shlosman, Begelman \& Frank 1990; Combes et al. 1994; Rosario et al. 2018).

Star formation tracers in the optical spectral range, for example, are considerably well known and have been used to identify star formation in galaxies over the years (e.g. Bica 1988; Kennicutt 1988; Worthey \& Ottaviani 1997; Gu et al. 2006). Over the past two 
decades, optical studies on scales of hundreds of parsecs around the nucleus of Seyfert galaxies have shown that in $\sim 40$ per cent of them, young stars $(t \lesssim 50 \mathrm{Myr})$ coexist with the AGN (e.g. StorchiBergmann et al. 2000, 2001; González Delgado, Heckman \& Leitherer 2001; Cid Fernandes et al. 2004; Asari et al. 2007; Dors et al. 2008), supporting the so-called AGN-starburst connection (Shlosman, Frank \& Begelman 1989; Shlosman et al. 1990; Heckman et al. 1997). Moreover, these studies suggest that the main difference between the stellar population of active and non-active galaxies is an excess of intermediate-age stars $(t \sim 0.05-2 \mathrm{Gyr})$ in the former.

Similar results were found in stellar population studies using nearinfrared (NIR) long-slit spectroscopy (Riffel et al. 2007, 2009b; Martins et al. 2010), suggesting that the continuum of Seyfert is dominated by the contribution of intermediate-age stellar populations. Moreover, contribution of hot dust emission to the nuclear NIR spectra was found in 50 percent of Seyfert 1 and in 20 per cent of Seyfert 2 sources (Rodríguez-Ardila, Contini \& Viegas 2005; Rodríguez-Ardila \& Mazzalay 2006; Riffel et al. 2009a,b; Riffel, Storchi-Bergmann \& McGregor 2009c). The fact that the NIR is less affected by dust than the optical bands makes this spectral range the most suitable one to unveil the nuclear stellar populations in highly obscured sources (Origlia \& Oliva 2000), such as the case of Seyfert 2 galaxies. The observed properties of this subclass of AGN suggest the SMBH powering the AGN is obscured to the line of sight by the torus surrounding the central engine. In addition, as has already been shown by e.g. Davies et al. (2006), star formation can take place within the torus, on scales of tens of parsecs from the nucleus.

The advent of James Webb Space Telescope (JWST) and the near- and mid-infrared integral field spectroscopic capabilities it will provide open the study of spatially resolved obscured stellar populations and hot dust using the entire $0.6-28 \mu \mathrm{m}$ range. Thus, to test the stellar population synthesis method in this particular spectral range is of fundamental importance for forthcoming studies with JWST.

The AGNIFS (AGN Integral Field Spectroscopy) team has started to characterize the stellar population in the inner kiloparsecs of a sample of nearby Seyfert galaxies (Mrk 1066, Mrk 1157, NGC 1068, NGC 5548, and Mrk 573) using Gemini Near-Infrared Integral Field Spectrograph (NIFS). For Mrk 1066, Mrk 1157, and Mrk 573, the inner $\sim 200 \mathrm{pc}$ are dominated by old stars ( $t \geq 2 \mathrm{Gyr}$ ), while intermediate-age stars $(0.3 \leq t \leq 0.7 \mathrm{Gyr})$ are the dominant contributors to the circumnuclear rings found in these sources (Riffel et al. 2010, 2011c; Diniz et al. 2017). These circumnuclear rings of intermediate-age stars are correlated with low stellar velocity dispersion values $\left(\sigma_{\star} \sim 50 \mathrm{kms}^{-1}\right)$, being consistent with a scenario in which the origin of the low $-\sigma_{\star}$ rings is a past event that triggered an inflow of gas and formed stars that still keep the colder kinematics of the gas from which they formed. StorchiBergmann et al. (2012) found two recent episodes of star formation in NGC 1068: a first one that took place $300 \mathrm{Myr}$ ago extending over the inner $300 \mathrm{pc}$ of the galaxy and a second one that occurred just $30 \mathrm{Myr}$ ago in a ring-like structure at $\sim 100 \mathrm{pc}$ from the nucleus, where it is coincident with an expanding ring of warm $\mathrm{H}_{2}$ emission. Schönell et al. (2017), on the other hand, detected a dominant intermediate-age stellar population component (SPC) in the inner $160 \mathrm{pc}$ of NGC 5548, while an old (>2 Gyr) SPC dominates the region between 160 and $300 \mathrm{pc}$. Dust emission has been detected in all sources (with exception of NGC 1068) accounting for $30-90$ per cent of the $K$-band nuclear flux, while a featureless continuum component associated with the AGN emission was detected in three sources, contributing with $\sim 20-60$ per cent of the $K$-band nuclear flux (NGC 1068, Mrk 573, and NGC 5548).

Modeling the $\mathrm{Br} \gamma$ equivalent width (EW), supernovae rate, and mass-to-light ratio, Davies et al. (2007) have quantified the SFH in the centre of nine nearby Seyfert galaxies using their STARS code. Their results indicate the age of the stars that contributes most to the NIR continuum lie in the range 10-30 Myr, pointing out that these ages should be considered only as 'characteristic', as they have not performed a proper spectral synthesis, suggesting that there may be simultaneously two or more stellar populations that are not coeval.

All these previous studies have focused on either nearby luminous Seyfert galaxies, in which the output energy is dominated by the AGN, or on galaxies with luminous circumnuclear star-forming rings. Low-luminosity AGNs (LLAGNs), on the other hand, including low-luminosity Seyfert galaxies, classical LINERs, weak-[O I] LINERs, and LINER/H II transition-like objects are the most common type of galaxies that display nuclear activity (for a review on LINERs, see Filippenko 2003; Singh et al. 2013; Belfiore et al. 2016; Hsieh et al. 2017). LINERs alone comprise 50-70 per cent of AGNs and 20-30 percent of all galaxies in surveys of nearby bright galaxies (Ho et al. 1997). Therefore, to identify the nature of the energy source in LLAGNs, as well as to disentangle the contribution of both SPCs and accreting black holes to the energy output of these sources is of utmost importance. In this context, a key object to move forward on the understanding of the role played by star formation in LLAGNs is NGC 4303, a nearby LINER/Seyfert 2 (Filippenko \& Sargent 1986; Kennicutt, Keel \& Blaha 1989; Ho et al. 1997; Colina \& Arribas 1999) galaxy with an LLAGN coexisting with a young massive star cluster in the nucleus and a star-forming circumnuclear ring (see Section 1.1).

\subsection{Previous stellar population studies of NGC4303}

NGC 4303 is a barred spiral classified as SB(rs)bc (de Vaucouleurs et al. 1991), located in the Virgo Cluster, at a distance of $16.1 \mathrm{Mpc}$ (Ferrarese et al. 1996). A nuclear UV-bright spiral arm with an outer radius of $225 \mathrm{pc}$ and spiraling all the way down to the unresolved (size $<8 \mathrm{pc}$ ) UV-bright core was detected in the HST/WFPC2 F218W images (Colina et al. 1997). This spiral structure was traced by several distinct regions, identified as young massive stellar clusters. These authors concluded that the unresolved LINER-like core contributes with 16 percent of the UV luminosity of NGC 4303, which is dominated by the massive star-forming regions. Another important result is the presence of spiral star-forming structure in the nuclear region of a barred spiral supports the bar-induced AGNstarburst scenario. In this sense, the gas in the bar-driven nuclear spiral can flow inward towards the core and fuel a pre-existing black hole, producing an AGN (Shlosman et al. 1990; Combes 2003).

In a later work, Colina \& Arribas (1999) used optical integral field spectroscopy to study the inner $9 \operatorname{arcsec} \times 8 \operatorname{arcsec}$ (i.e. $700 \times 620 \mathrm{pc}^{2}$ ) and found, from optical emission-line ratios, that the circumnuclear star-forming regions (CNSFRs) of this source have ages of 2-3 Myr (extremely young clusters of massive stars). Also, they concluded the presence of a massive $\left(8 \times 10^{4} \mathrm{M}_{\odot}\right)$ and young (3.5-4 Myr) stellar cluster in the nucleus is consistent with the observed properties of the core of NGC 4303 (e.g. optical emissionline ratios, $\mathrm{UV}$, and $\mathrm{H} \alpha$ luminosities). Nevertheless, the presence of a non-thermal power-law AGN-like ionizing source cannot be ruled out. Thus, the authors classified the core of this galaxy as a [O I]-weak LINER or low-excitation Seyfert 2.

Studying the radial distributions of the $\mathrm{Mg}_{2}$ and Fe5270 Lick spectral indices in the disc of NGC 4303, Mollá, Hardy \& 
Beauchamp (1999) found while in NGC 4303 both indices steeply rise towards the central region $(\lesssim 2 \mathrm{kpc})$, in the other objects studied by them (NGC 4321 and NGC 4535) a central dip is observed. In their study, by using simple stellar population (SSP) models, these authors inferred that NGC 4303 is still forming stars.

Using WFPC2 (F218W, F606W) and NICMOS (F160W) Hubble Space Telescope (HST) images, Colina \& Wada (2000, hereafter C00) studied the inner 300 pc of NGC 4303 and identified a nuclear elongated bar-like structure of $250 \mathrm{pc}$ in size. The images revealed a complex gas/dust distribution with a two-arm spiral structure of about $225 \mathrm{pc}$ in radius. Also, they estimated the age of the UV-bright knots located along the star-forming spiral structure as 5-25 Myr and masses of $0.5 \times 10^{5}-1 \times 10^{5} \mathrm{M}_{\odot}$.

From UV imaging and spectroscopy (HST/STIS), Colina et al. (2002, hereafter C02) concluded the UV emission from the nucleus of NGC 4303 comes from a region of $3.1 \mathrm{pc}$ and it is identified as a young $(4 \mathrm{Myr})$, massive $\left(10^{5} \mathrm{M}_{\odot}\right)$ nuclear cluster. The authors found that this compact super-star cluster (SSC), commonly detected in the (circum)nuclear regions of spirals and starburst galaxies, is the dominant ionizing source in the nucleus. According to this study, an additional non-thermal ionizing source due to an AGN is not required. They also discuss the possibility of having an intermediate/old (1-5 Gyr) star cluster coexisting with the low efficiency accreting black hole and the young and luminous SSC. Jiménez-Bailón et al. (2003) analyzed 2-10 keV observations from the Chandra X-ray satellite and indicated that an additional compact source was required to explain the $1.5-5 \mathrm{keV}$ emission, possibly a low-luminosity AGN.

In Riffel et al. (2016, hereafter R16), we presented the results concerning the kinematics and excitation properties of the different phases of the interstellar medium in the circumnuclear region of NGC 4303 using the same SINFONI datacubes presented in this work. A circumnuclear ring of star-forming regions $(r \sim$ $200-250 \mathrm{pc}$ ) was detected, displaying young ages in the range $2.5-$ 15 Myr. Star formation in the ring appears to be episodic, with stars forming quasi-simultaneously. Moreover, NIR emission-line ratios $\left([\mathrm{Fe} \mathrm{II}] / \mathrm{Br} \gamma\right.$ and $\left.\mathrm{H}_{2} / \mathrm{Br} \gamma\right)$ are consistent with the presence of an $\mathrm{AGN}$ and/or an $\mathrm{SN}$-dominated star-forming region in the core (inner $60 \mathrm{pc}$ radius) of NGC 4303.

Here we perform, for the first time, a spatially resolved full spectral fitting stellar population study of the nuclear region $(\sim 200 \mathrm{pc}$ radius) of NGC 4303, using both STARLIGHT code and VLT/SINFONI data. This paper is structured as follows. Section 2 presents the observations and data-reduction procedures, while in Section 3 we introduce the stellar population synthesis method used in this work. The results are presented in Section 4 and discussed in Section 5. Finally we present our conclusions in Section 6. North is up and east is to the left throughout the images and maps presented in this paper.

\section{OBSERVATIONS AND DATA REDUCTION}

The data used are the same as the datacubes presented in R16. Here we summarize the information on observations and data-reduction process, as follows. The observations were done during the period 82B (2009 February) at the ESO Very Large Telescope (VLT) with SINFONI, a NIR integral field spectrograph (Eisenhauer et al. 2003; Bonnet et al. 2004). The pointings were centered on the nucleus of the galaxy, covering a field of view (FoV) of $\sim 8 \times 8$ arcsec per exposure, enlarged by dithering up to $\sim 9^{\prime \prime} .25 \times 9$ '.25, with a plate scale of $0^{\prime \prime} .125 \times 0^{\prime \prime} .250 \mathrm{pixel}^{-1}$. The final data cube was re-sampled to a scale of $0^{\prime} .125 \times 0$ '. $125 \mathrm{pixel}^{-1}$, corresponding to a spatial sampling of $\sim 10 \mathrm{pc}$ per spaxel.

The data were taken in the $J(1.10-1.35 \mu \mathrm{m}), H(1.45-1.80 \mu \mathrm{m})$, and $K(1.97-2.44 \mu \mathrm{m})$ bands with a total integration time of $2400 \mathrm{~s}$ per band. In the same way, a set of photometric standard stars was observed to perform the telluric and flux calibration. We estimated the spatial resolution of our seeing-limited observations by fitting a 2D Gaussian profile to a collapsed image of the standard stars. The spatial resolution (FWHM) measured for each band is $\sim 1^{\prime \prime}$, $\sim 0$ '. 6 , and $\sim 0^{\prime \prime} .5$ for $J, H$, and $K$ bands, respectively, that correspond to $78 \mathrm{pc}, 47 \mathrm{pc}$, and $39 \mathrm{pc}$, respectively, at the adopted distance of 16.1 Mpc for NGC 4303. The reduction and calibration processes were performed using the standard ESO pipeline ESOREX (version 3.8.3), and our own IDL routines (see R16, where a complete description of the reduction and calibration procedures is provided).

\subsection{Relative flux calibration}

The accurate determination of the continuum shape plays an important role on the stellar population determinations (Baldwin et al. 2018). The stellar population synthesis technique used in this work is strongly dependent on a reliable continuum fit, meaning that any problem in the relative flux calibration between spectral bands would compromise the results. When dealing with emission-line measurements, on the other hand, this issue is much less important. The continuum estimation needed to fit an emission line is done within the range of only one spectral band, being nearly independent of relative flux calibrations. With this in mind, we decided to add an extra step concerning the relative flux calibration of our data set, in order to get a reliable relative calibration, adequate for the purpose of spectral energy distribution fitting.

Our target was observed in three different nights and only a limited number of standard stars were available to perform the flux calibration. Although the efficiency curves appear to be consistent, the flux calibration of the $J$-band data might be inaccurate outside the $1.15-1.30 \mu \mathrm{m}$ range, since the slope of the curves is very uncertain beyond this limit and the spectra is completely dominated by the noise from the atmospheric absorption bands. This fact is translated to uncertainties on the slope of the spectra in the data cubes (DCs) which, thus, cannot be used to perform a reliable stellar population synthesis using full spectral fitting (see Cid Fernandes et al. 2005b; Baldwin et al. 2018).

To address that, we used NIR cross-dispersed data of NGC 4303 from Martins et al. (2013a), obtained at the NASA 3m Infrared Telescope Facility (IRTF) using the SpeX spectrograph. As the observations at the $J, H$, and $K$ bands are done simultaneously in the cross-dispersed mode, the data do not suffer from relative flux calibration problems. Therefore, assuming that the shape of the SpeX data was correct, we used it to scale our SINFONI observations. It is worth mentioning that we have used SINFONI integrated spectra extracted with the same aperture size and position angle as the SpeX data.

\subsection{Instrumental fingerprint removal}

The instrumental fingerprint removal was performed using the Principal Component Analysis (PCA) Tomography technique, which consists of applying PCA to data cubes. First, the spectral lines of the data cube were removed. Then, PCA was applied and the obtained eigenvectors related to the fingerprint were used to construct a data cube containing only the fingerprint. Such a data cube was subtracted from the original one, completing the removal of the 
instrumental artifact. This entire procedure was applied separately to the $J, H$, and $K$ bands of the data cube. For more details, see Menezes, Steiner \& Ricci (2014) and Menezes et al. (2015).

\section{STELLAR POPULATION SYNTHESIS METHOD}

A common way to disentangle the spectral energy distribution components of a galaxy spectrum is by performing stellar population synthesis. This method consists in fitting the galaxy absorption and continuum spectrum with a combination of SSP components. Therefore, the two main ingredients are (i) the SSP templates (hereafter, base set) and (ii) the fitting code.

\subsection{Base set}

An ideal set of templates should be able to foresee all the features expected to be found in spectra of galaxies (Schmidt et al. 1991; Cid Fernandes et al. 2005a). In other words, a reliable base set would be an empirical library of integrated spectra of star clusters (i.e. they only depend on ages and metallicities of the stars and are free from any assumptions on stellar evolution and the initial mass function [IMF]; Bica \& Alloin 1986; Riffel et al. 2011a). However, up to now there is no such library available in the literature for the NIR spectral region. Thus, the use of a base set composed of theoretical SSPs, covering this spectral region, has become a common approach (e.g. Riffel et al. 2009b; Martins et al. 2010; Dametto et al. 2014).

Since the NIR carries fingerprints from evolved stars (e.g. Riffel et al. 2007; Ramos Almeida, Pérez García \& Acosta-Pulido 2009; Martins et al. 2013b; Riffel et al. 2015) and these are crucial to model the absorption line spectra of the galaxies, it is important to make use of SSPs models that can predict these features. Following Dametto et al. (2014), we decided to used the Maraston (2005, hereafter M05) ${ }^{1}$ Evolutionary Population Synthesis (EPS) models, which include empirical spectra of C- and O-rich stars (Lançon \& Wood 2000) and thus are able to predict these features. We also tested other SSPs models from Bruzual \& Charlot (2003, hereafter BC03) and Conroy, Gunn \& White (2009, hereafter C09); see Section 4.1 for this discussion.

The M05 models span an age range from $0.001 \mathrm{Myr}$ to $15 \mathrm{Gyr}$ according to a grid of 67 models with six different metallicities $(0.005 \leq \mathrm{Z} / \mathrm{Z} \odot \leq 3.5), 2 \mathrm{IMFs}$ (Salpeter and Kroupa), and three horizontal branch morphologies (red, intermediate, or blue; for more details, see M05). It is worth noting the models with $Z=0.005 \mathrm{Z} \odot$ and $Z=3.5 \mathrm{Z} \odot$ are provided only for ages older than $1 \mathrm{Gyr}$ (in a grid of 16 ages) and are based on Cassisi (Cassisi, Castellani \& Castellani 1997b; Cassisi, degl'Innocenti \& Salaris 1997a) and Padova 2000 (Girardi et al. 2000) isochrones, respectively. The remaining four metallicities (computed for the full grid of 67 ages) are associated with Cassisi + Geneva (Schaller et al. 1992) tracks. The stellar spectra were taken from the BaSeL 2.2 library (Lejeune, Cuisinier \& Buser 1997, 1998), covering the spectral range of $91 \AA$ to $160 \mu \mathrm{m}$, with a spectral resolution of $5-10 \AA$ up to the optical region, and 20-100 $\AA$ in the near- and far-infrared.

The BaSeL 2.2 is a library of low-resolution stellar spectra based on the theoretical templates compiled by Lejeune et al. (1997, 1998). This library is widely used in stellar population synthesis studies and was constructed by combining the model atmosphere spectra of

\footnotetext{
${ }^{1}$ Available at http://www.icg.port.ac.uk/ maraston/Claudia's_ Stellar_Pop ulation_Model.html
}

Bessell et al. (1989, 1991) with the models for cool stars by Fluks et al. (1994). As synthetic spectral libraries do not contain TPAGB carbon- and oxygen-rich stars, empirical and time-averaged spectra of C- and O-type stars from Lançon \& Mouhcine (2002) were included in the M05 models.

In order to avoid redundant information and degeneracies in the base set, we used only the representative SSPs (see Dametto et al. 2014 for further details) and ended up with a final base set composed as follows: 31 ages $(1.0 \mathrm{Myr} \leq t \leq 13.0 \mathrm{Gyr})$ for each of the four metallicities $\left(Z=0.02,0.5,1\right.$, and $\left.2 \mathrm{Z}_{\odot}\right)$ totaling 124 SSPs. We also included blackbody functions for temperatures in the range $700-1400 \mathrm{~K}$ in steps of $100 \mathrm{~K}$ and a power law $\left(F_{v} \propto v^{-1.5}\right)$ in order to account for possible contributions from dust emission and from a featureless continuum, respectively, at the nucleus (Cid Fernandes et al. 2004; Riffel et al. 2009b).

The spectral resolution of M05 models in the NIR is significantly lower $(R \leq 250)$ than that of the observed data $(R \sim 2000)$ and varies with wavelength. For this reason, observations were degraded to the models' resolution by convolving them with a Gaussian.

\subsection{Fitting code}

Other fundamental ingredient in stellar population fitting is the code. Following Dametto et al. (2014), we used STARLIGHT code (Cid Fernandes et al. 2005b; Mateus et al. 2006), which fits the observed spectrum $O_{\lambda}$ with a combination in different proportions of $N_{\star}$ SSPs in the base set $-b_{\mathrm{j}, \lambda}-$ taken from the EPS models. One of the key features of STARLIGHT is that the code fits the entire spectrum (from 0.8 to $2.4 \mu \mathrm{m}$ in this case), excluding emission lines and spurious features (e.g. cosmic rays, and telluric regions), which are masked out.

Basically, STARLIGHT solves the following equation for a model spectrum $M_{\lambda}$ (Cid Fernandes et al. 2005a):

$M_{\lambda}=M_{\lambda 0}\left[\sum_{j=1}^{N_{\star}} x_{j} b_{j, \lambda} r_{\lambda}\right] \otimes G\left(v_{\star}, \sigma_{\star}\right)$,

where $M_{\lambda o}$ is the synthetic flux at the normalization wavelength $\left(\lambda_{0}=2.067 \mu \mathrm{m}\right) ; x_{j}$ is the $j$ th population vector component of the base set; $b_{j, \lambda} r_{\lambda}$ is the reddened spectrum of the $j$ th SSP normalized at $\lambda_{0}$ in which $r_{\lambda}=10^{-0.4\left(A_{\lambda}-A_{\lambda 0}\right)}$ is the extinction term; $\otimes$ denotes the convolution operator, and $G\left(v_{\star}, \sigma_{\star}\right)$ is the gaussian distribution used to model the line-of-sight stellar motions, centered at velocity $v_{\star}$ with dispersion $\sigma_{\star}$. We choose as normalization wavelength $\lambda_{0}=2.067 \mu \mathrm{m}$, since $K$-band spectra present a higher SNR than those in the $J$ and $H$ bands, and the spectral region near $2.067 \mu \mathrm{m}$ is free from emission/absorption lines.

Velocity dispersion is a free parameter for STARLIGHT, which broadens the SSPs in order to better fit the absorption lines in the observed spectra, however this step is not relevant in our case. Assuming M05 (BaSeL based models) spectral resolution in velocity units as $\sim 1500 \mathrm{~km} \mathrm{~s}^{-1}$, the velocity dispersion is $\sim 640 \mathrm{~km} \mathrm{~s}^{-1}$, which are both much higher values than those calculated in R16 $\left(v_{\star}:-80 /+80 \mathrm{~km} \mathrm{~s}^{-1}\right.$ and $\sigma_{\star}: 20 / 100 \mathrm{~km} \mathrm{~s}^{-1}$ ) for the stellar kinematics. Thus, because of the models' low resolution, we kept the kinematic parameters fixed $\left(v_{\star}=0.0\right.$ and $\left.\sigma_{\star}=640 \mathrm{~km} \mathrm{~s}^{-1}\right)$ during the fits. It is important to highlight that for the low-resolution models, the age information is encoded in the continuum shape (Riffel et al. 2009b; Baldwin et al. 2018; Dahmer-Hahn et al. 2018).

The extinction law used in this work was that of Calzetti law (Calzetti et al. 2000) implemented by Hyperz (Bolzonella, 

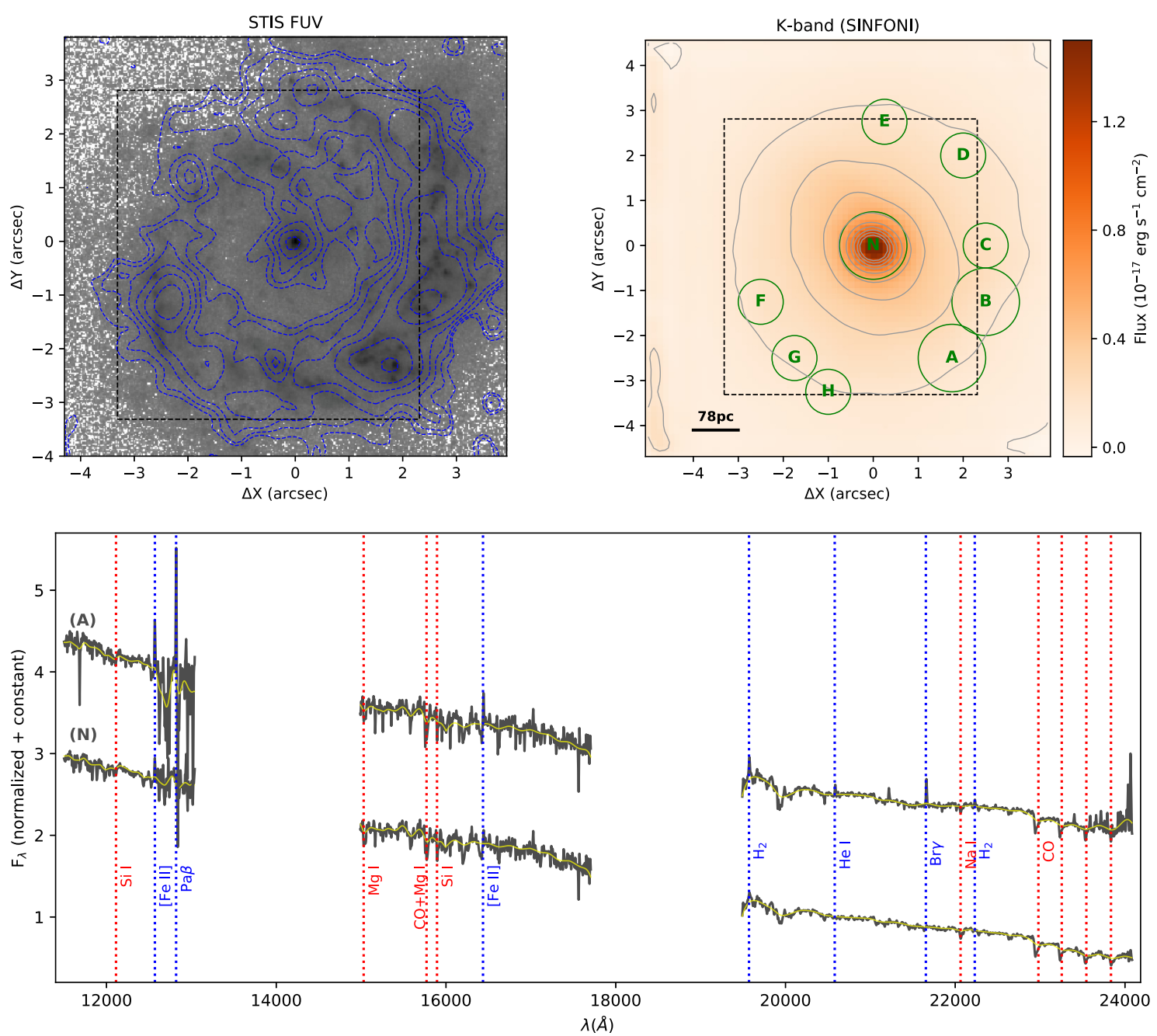

Figure 1. STIS (Space Telescope Imaging Spectrograph) F25QTZ far ultraviolet image of NGC 4303 previously presented by C02 in gray scale with Br $\gamma$ emission line (SINFONI) contours in blue (top left). $K$-band continuum (contours are shown in grey to help visualization), reconstructed from the SINFONI data cube as an average of the fluxes between 2.22 and $2.27 \mu \mathrm{m}$ (top right). Green circles mark the position of the CNSFRs previously reported by R16 and the black box in both images denotes the FoV used in this work. The bottom panel shows the near-IR spectra for the nucleus (bottom) and for position A (top), extracted with a circular aperture with radius 0.75 and normalized at $2.067 \mu \mathrm{m}$. The smoothed spectra are overplotted in yellow. A constant (1.5) was added to the spectrum of Region (A) for visualization purpose. Absorption (red) and emission (blue) lines are marked.

Miralles \& Pelló 2000), a public photometric redshift code that computed the Calzetti extinction law for $\lambda>2.2 \mu \mathrm{m}$.

Lastly, the code searches for the minimum of the equation:

$\chi^{2}=\sum_{\lambda}\left[\left(O_{\lambda}-M_{\lambda}\right) w_{\lambda}\right]^{2}$

and the best fit is achieved. In order to measure the robustness of the stellar population fit, we can use STARLIGHT output parameters $\chi^{2}$ and Adev. The later is the per cent mean deviation $\left|O_{\lambda}-M_{\lambda}\right| / O_{\lambda}$, where $O_{\lambda}$ is the observed spectrum and $M_{\lambda}$ is the fitted model. Emission lines and spurious features (telluric regions, cosmic rays) are masked out by using $w_{\lambda}=0$ in the regions where they are located. This procedure is done by first constructing a general mask, based on the emission-lines position. Next, we inspect spaxel-byspaxel to remove any additional spurious data. For more details, see Dametto et al. (2014) and STARLIGHT manual available at http: //www.starlight.ufsc.br.

\section{RESULTS}

Following Dametto et al. (2014, and references therein), the stellar population vectors have been binned in three main components: young (blue): $x_{y}\left(t \leq 50 \times 10^{6} \mathrm{yr}\right)$, intermediate-age (orange): $x_{i}$ $\left(50 \times 10^{6}<t \leq 2 \times 10^{9} \mathrm{yr}\right)$, and old (red): $x_{o}\left(t>2 \times 10^{9} \mathrm{yr}\right)$. An example of the final fit for the nuclear region $(r=0$ '. $75 \sim 60 \mathrm{pc}$, central green circle in Fig. 1) of NGC 4303 is presented in Fig. 2. The nuclear spectrum is well described by a series of star formation bursts, the first one occurring $\sim 13 \mathrm{Gyr}$ ago, ${ }^{2}$ contributing with $\sim 50$ per cent of the flux at $2.067 \mu \mathrm{m}$. The fit reproduces individual minor bursts with ages ranging from 0.3 to $0.7 \mathrm{Gyr}$, corresponding to a 15 per cent contribution of the intermediate-age SPC, while the young SPC accounts for 35 percent of the flux, with a major burst at 7.5 Myr ago. No contribution of the featureless continuum and/or

\footnotetext{
${ }^{2}$ Note that the base is not a continuous distribution of ages, with the old ages being represented by $13 \mathrm{Gyr}$ SSPs in this case; for details, see (Dametto et al. 2014).
} 

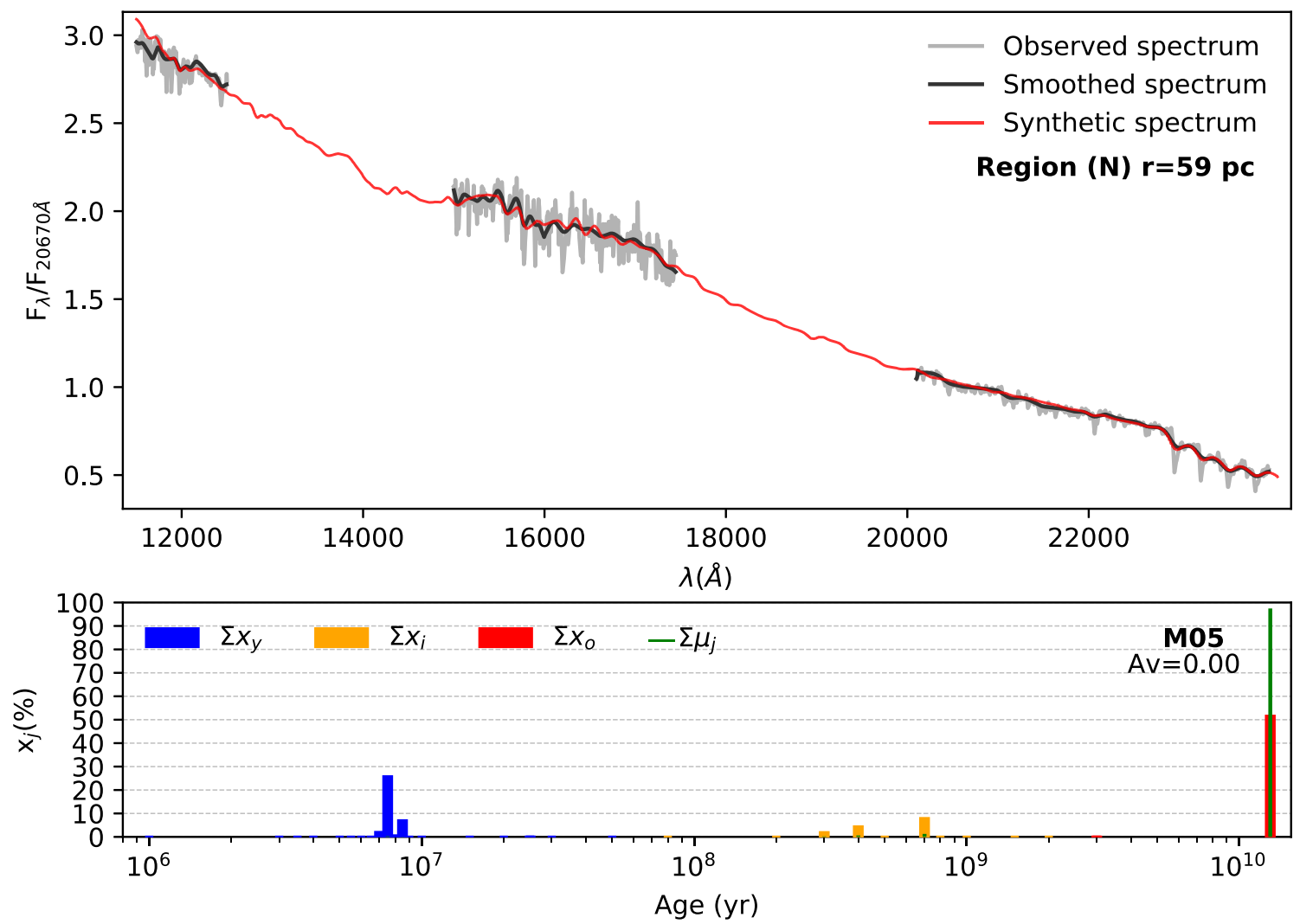

Figure 2. Example of the stellar population synthesis results for the nuclear region ( $r=0^{\prime \prime} 75$, corresponding to $60 \mathrm{pc}$ ) of NGC 4303 (central green circle in the maps). Top panel: observed (gray), smoothed (black), and synthetic (red) spectrum, normalized to unit at $2.067 \mu \mathrm{m}$. Bottom panel: Histogram displaying the flux ( $x_{j}$, coloured bars) and mass-weighted $\left(\mu_{j}\right.$, green lines) stellar population vectors contributions sorted only by age (metallicities summed) and colour coded by the three SPC age bins: young (blue: $\leq 50 \mathrm{Myr}$ ), intermediate-age (orange: $0.05-2 \mathrm{Gyr}$ ) and old (red: $>2 \mathrm{Gyr}$ ). The reddening value (in magnitude units) is also shown. Telluric absorption regions are omitted.

hot dust components was necessary in order to reproduce the nuclear continuum of this source. Green lines represent the percentage contribution in mass of each SSP. The old SPC $\left(m_{o}\right)$ dominates the mass contribution with 98 percent, while the contribution of the other two components is negligible. Moreover, the code fits a dustfree $\left(A_{V}=0.0\right)$ spectrum for the inner $60 \mathrm{pc}$ of NGC 4303, agreeing with previous results from $\mathrm{C} 02$, which found low extinction values $\left(A_{V}=0.3 \mathrm{mag}\right)$ for the inner 0.9 of this source.

Spatial distribution of the percent flux (top panels) and mass (bottom panels) contribution of each SPC bin is shown in Fig. 3. ${ }^{3}$ As for Fig. 1, green circles represent the CNSFRs analyzed in R16. The physical sizes ${ }^{4}$ of these regions are Regions N/A/B $(r=0$ '.75$60 \mathrm{pc})$ and others $(r=0,5-39 \mathrm{pc})$. We point out that the FoV used in this work is smaller than that used to study the emission lines in $\mathrm{R} 16$, mainly due to problems with the relative flux calibration and due to low SNR close to the borders, which represents a limitation when performing stellar population synthesis (Cid Fernandes et al. 2005b).

Analyzing the SPC contributions in Fig. 3, we can see the age of the dominant stellar population presents a spatial variation, sug-

\footnotetext{
${ }^{3}$ We smoothed all maps to the $H$-band spatial resolution $(0.6)$. Note that the $K$-band has a higher (0.'5) spatial resolution, while the $J$-band has a lower one ( $\left.1^{\prime \prime}\right)$. We decided to smooth the maps, using the $H$-band resolution, since we are using a small spectral region in the $J$-band to perform the fits.

${ }^{4}$ The apertures were chosen by the authors in R16 to be larger than the seeing ( $\left.\sim 0^{\prime \prime} 5\right)$ and to include most of the $\mathrm{Br} \gamma$ flux of each region.
}

gesting an age stratification along the inner $\sim 200 \mathrm{pc}$ radius of this source. Three main features are evident from the maps: A blob dominated by young stars in the nuclear region shifted towards east from the centre (here defined as the peak of the $\mathrm{Br} \gamma$ emission line) and the UV emission peak; a second blob dominated by intermediate-age stars located southwest from the centre, clearly seen in the middle panels; and a more internal arm-like structure very close to the blob of young stars, mainly seen in the $m_{i}$ map.

The youngest SPCs $\left(x_{y}\right.$ and $\left.x_{i}\right)$ are distributed along the circumnuclear region (200-250 inner parsecs), similar to the results predicted from the emission line gas presented in R16. It is important to highlight that our FoV does not cover the whole western part of the circumnuclear ring (we miss most of regions $B$ and $C$ ). In fact, a major contribution of the young SPC is seen in the northeastern area, which is co-spatial with dustier regions reported by $(\mathrm{C} 00$, see their fig. 3) using $V-H$ colour. As we are using NIR data, we were able to penetrate deeper into the dust layers, accessing these young stars missed in the UV and optical ranges, which are more sensitive to dust obscuration. This could explain why the young SPC map is not tracing the spiral-arm structure clearly seen in the UV emission (Fig. 1, darker knots represent less obscured regions), but the circumnuclear ring. The old SPC is distributed outside the two blob structures, being more prominent northwest from the centre.

Flux-weighted results have a dependence on the choice of the normalization wavelength (Riffel et al. 2011b). Thus, one should take this into consideration when comparing results from different spectral regions. By using the knowledge of stellar evolution, one 

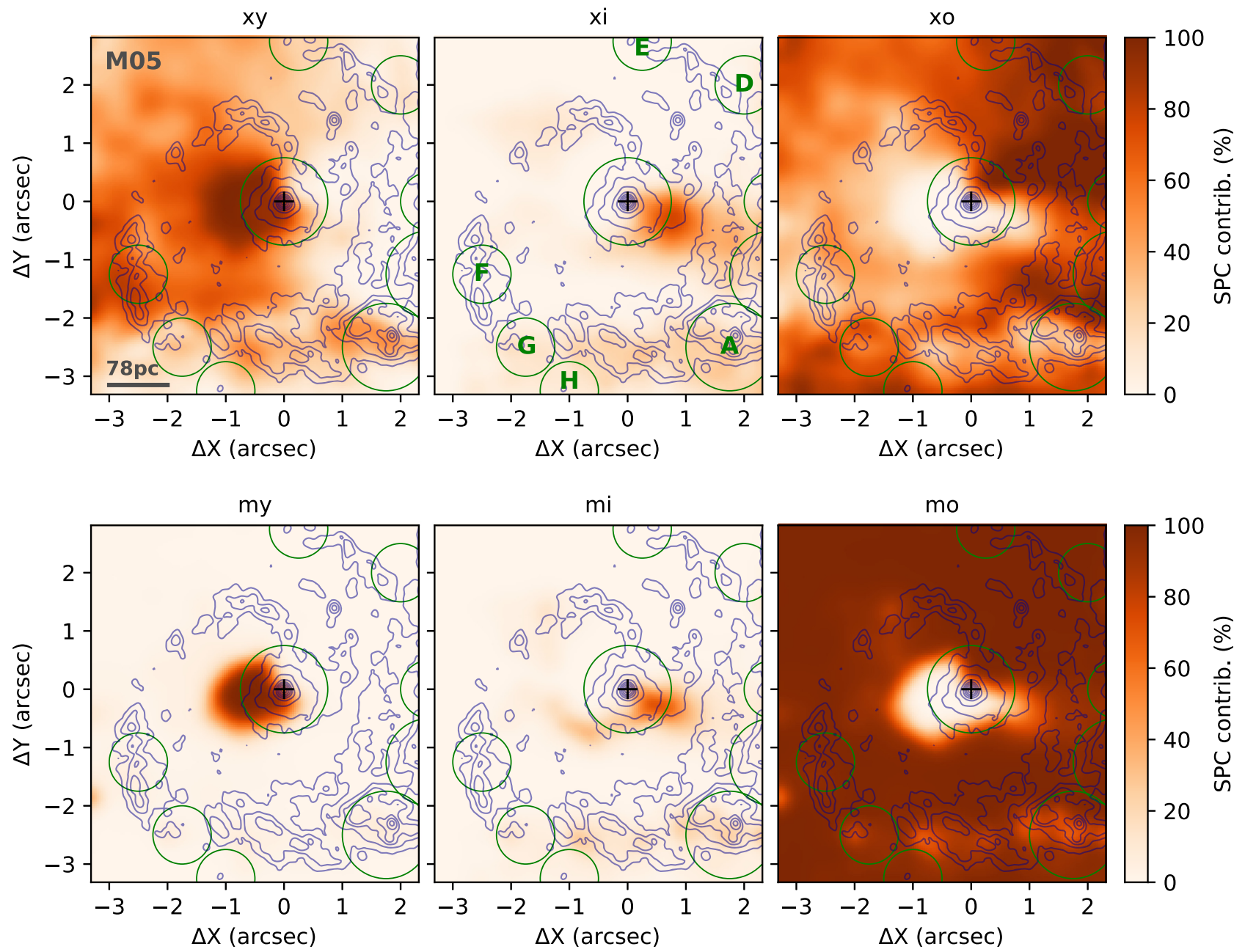

Figure 3. Results using M05 models. Spatial distribution of the percent contribution of each SPC to the flux ( $x_{j}$, top panels) and mass ( $m_{j}$, bottom panels), where $j$ represents the age of the SPC: young (y: $\leq 50 \mathrm{Myr}$ ), intermediate-age (i: $0.05-2 \mathrm{Gyr}$ ), and old (o: > 2 Gyr). Green circles mark the CNSFRs, clearly seen in the EW Br $\gamma$ previously reported by R16. The black cross represents the peak of the Br $\gamma$ emission line. STIS far-ultraviolet broad-band image is shown in blue contours, tracing the spiral structure reported in $\mathrm{C} 02$.

can use the mass-to-light ratio (M/L) for each SSP and determine the percentage contribution in stellar mass, a physical parameter that does not depend on the normalization wavelength used in the fit. The bottom panels of Fig. 3 present the mass-weighted contribution of the SPCs. As we can see, the major contribution in mass comes from old ( $t>2 \mathrm{Gyr})$ stars. In addition, the three internal structures mentioned above (the two blobs and the inner spiral arm) are highlighted in these maps.

Besides the SPC distributions, STARLIGHT full spectral fitting provides a measure of the internal extinction $\left(\mathrm{A}_{V}\right)$, which is shown in the left-hand panel of Fig. 4. The nuclear spaxels clearly display the highest values $\left(\mathrm{A}_{V}=2.3 \mathrm{mag}\right)$, within scales of tens of parsecs, while the average value over the FoV is $\sim 0.3 \mathrm{mag}$. The southern region of the reddening map displays a stripe-like pattern with reddening values around $1.5 \mathrm{mag}$. This stripe-like pattern may be partially related with a residual 'instrumental fingerprint', which we were not able to remove completely (see Section 2.2), thus, possibly affecting the reddening values in these locations.

STARLIGHT code also outputs the mass that has been processed into stars over the last $t$ years $\left(\mathbf{M}_{\star}^{t}\right)$. This can be used to estimate the mean SFR over a period of time $t$. We have estimated the mean $\mathrm{SFR}_{\star}$ over the last $10 \mathrm{Myr}$ as being the ratio of $\mathrm{M}_{\star}^{t} / t$ (for $t \leq 10 \mathrm{Myr}$ ), and it is presented in the middle panel of Fig. 4 . The nucleus clearly stands up with an SFR of $\sim 0.2 \mathrm{M}_{\odot} \mathrm{yr}^{-1}$ in the region co-spatial with the $A_{V}$ peak ( $\sim 40 \mathrm{pc}$ radius). The mean SFR over the whole FoV is $2.1_{-2.1}^{+9.7} \times 10^{-3} \mathrm{M}_{\odot} \mathrm{yr}^{-1}$.

As mentioned in Section 3, the quality of the fit can be measured by the STARLIGHT output parameter called per cent mean deviation: Adev $\left(\left|O_{\lambda}-M_{\lambda}\right| / O_{\lambda}\right)$, in which $O_{\lambda}$ is the observed spectrum and $M_{\lambda}$ is the fitted model (Cid Fernandes et al. 2004, 2005a). For our fits, Adev is below 4.5 per cent at most locations (see Fig. 4, righthand panel), indicating that the model reproduces well the observed spectra.

\subsection{Robustness of the stellar population results: comparison with BC03 and C09 SSP models}

In order to deepen our analysis, we decided to perform stellar population synthesis, using other EPS models available in the literature: Bruzual \& Charlot (2003, hereafter BC03) and Conroy et al. (2009, 

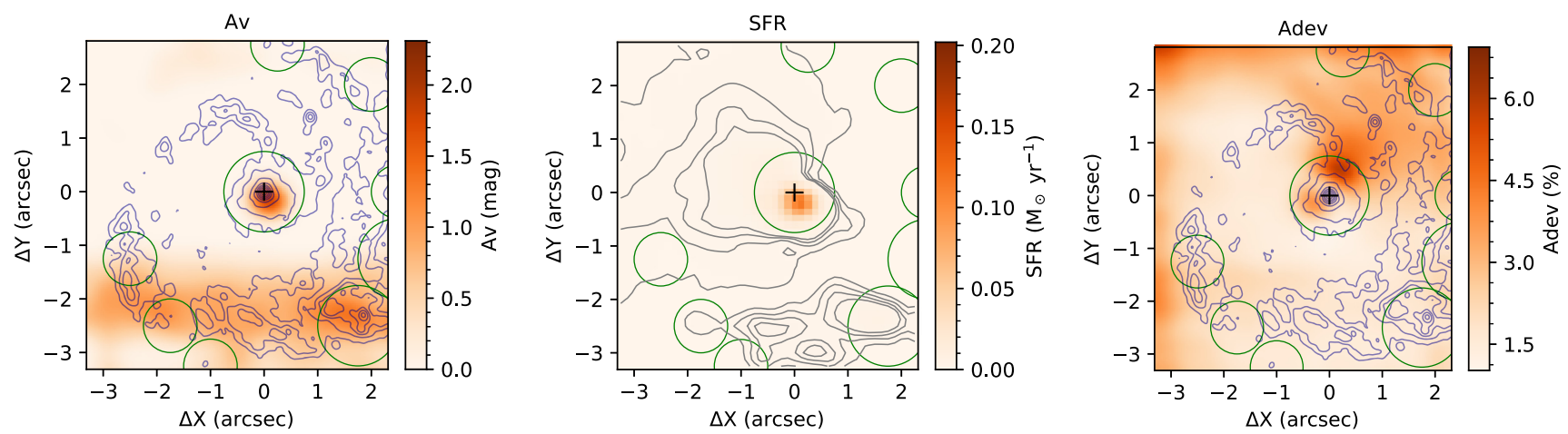

Figure 4. Left: Reddening $\left(\mathrm{A}_{V}\right)$; Middle: star formation rate (SFR) over the last $10 \mathrm{Myr}$; Right: Adev (per cent mean deviation from the spectral fit). Grey contours in the middle panel represent SFRs up to $2.5 \times 10^{-3} \mathrm{M}_{\odot} \mathrm{yr}^{-1}$, were included for display purposes.

hereafter C09). We do not include the models from Maraston \& Strömbäck (2011, hereafter M11) in this analysis, as the use of the lower resolution M05 models is a better option to deal with stellar population synthesis in the NIR when compared to the M11 ones (Dametto et al. 2014). Addressing the limitations on the M11 models, an example is the fact that above $\sim 1 \mu \mathrm{m}$ about half of the spectra (from Pickles stellar spectral library, used to construct the M11 models that extent to the NIR; Pickles 1998) lack spectroscopic observations leading the authors to construct a smooth energy distribution from broad-band photometry, which may imply that some NIR absorption features are not well resolved, even for these higher resolution models. New sets of models presented by Meneses-Goytia et al. (2015), Röck et al. (2016), and Conroy et al. (2018) are available, which make use of the IRTF and E-IRTF stellar libraries. However, the age range of these models $(t \geq 1 \mathrm{Gyr})$ is not adequate to fit the spectrum of galaxies with active star formation, therefore these models were not employed here.

First, the three models used in this work are constructed using different prescriptions (see Conroy 2013 for a review). Both BC03 and C09 models are constructed using the isochrone synthesis approach, while M05 uses the fuel consumption theory technique. In the former, the SSPs are constructed by integrating the contributions of all mass bins (along one isochrone) to the flux in the various passbands, after assuming an initial mass function (IMF). Yet, in the later, energetics of the post-main-sequence phases (i.e. the amount of fuel available for nuclear burning) are calculated using the evolutionary track of the turnoff mass.

The treatment of the TP-AGB phase-crucial to models the stellar populations in the NIR - is also a topic of discussion when comparing the results obtained with different EPS models. While BC03 constructed period-averaged spectra for C-type stars using broadband photometry to calibrate the low-resolution stellar templates of Höfner et al. (2000), both M05 and C09 include empirical spectra of carbon- and oxygen-rich stars from Lançon \& Mouhcine (2002). The inclusion of these empirical spectra of stars in the TP-AGB phase has enabled the detection of NIR characteristic absorption features, such as $\mathrm{TiO}(0.843$ and $0.886 \mu \mathrm{m})$, VO $(1.048 \mu \mathrm{m}), \mathrm{CN}$ (1.1 and $1.4 \mu \mathrm{m})$, and $\mathrm{CO}(1.6$ and $2.3 \mu \mathrm{m}$ ) bands (Riffel et al. 2015).

In recent studies, there has been an attempt to address discrepancies in stellar population synthesis results using different EPS models. Baldwin et al. (2018), studying a sample of 12 nearby early-type galaxies, recently concluded the variation in the derived SFHs using NIR is largely driven by the choice of stellar spectral library rather than the models' prescription. In order to better compare the results, our base sets are composed by SSPs constructed using basically the same stellar library $\left(\mathrm{BaSeL}^{5}\right)$ in the NIR spectral range.

Performing stellar population synthesis with STARLIGHT code for 7 spectra from early-type galaxies, Dahmer-Hahn et al. (2018) found systematic differences in the results using M05, BC03, and C09 models. While BC03 presented a higher contribution of young stellar populations, C09 displayed a major contribution of the older ages and M05 preferred solutions including a higher contribution (when compared to the other two models) of the intermediate-age components. One way to diminish such systematic effects is to fix the kinematic fit while running the code. As we degrade the resolution of the data to match the low resolution of these models in the NIR, the stellar features are considerably broadened, thus one cannot rely on the kinematic information obtained with STARLIGHT.

We have tested the results keeping the kinematics parameters fixed for NGC 4303 (see Section 3.2) and we were able to smooth the systematic effects found by Dahmer-Hahn et al. (2018), concluding the inclusion of a kinematic fit while running STARLIGHT with low-resolution models $(R \sim 300)$ do not yield reliable results, since the stellar features in the data are considerably broadened.

Base sets for $\mathrm{BC} 03$ and $\mathrm{C} 09$ were constructed in the same way as that of M05 and the same fitting procedure was applied (see Figs 5-7). C09 models do not provide the fraction of the initial stellar mass that is still present in the form of stars at the age $j$ for each base set component.Therefore we were not able to calculate the percentage mass contribution using these models and we only present the flux-weighted results.

In Fig. 5 we present an example of the fits for Region $A$ (see Fig. 1) using the three EPS models for comparison. From the top panel we can see the overall good quality of the fits, primarily in the $K$ band. Analyzing the histograms in the bottom panels, it is clear that the results assuming the binned stellar population vectors are in agreement between the models, presenting an $\sim 40$ percent contribution for both young (8-9 Gyr) and old (9/13 Gyr) SPC, while the remaining $\sim 20$ percent comes from intermediate-age stars (0.5-1 Gyr), with exception of BC03 results that do not display any contribution of the $x_{i}$ component and present an increase in $x_{y}$ ( 60 per cent).

The reddening solutions are similar, deviating at most in $0.17 \mathrm{mag}$, which can be explained by the fact that the NIR spec-

${ }^{5}$ M05: BaSeL 2.2 (Lejeune et al. 1997, 1998) and BC03/C09: BaSeL 3.1 (Lejeune et al. 1997, 1998; Westera et al. 2002) 

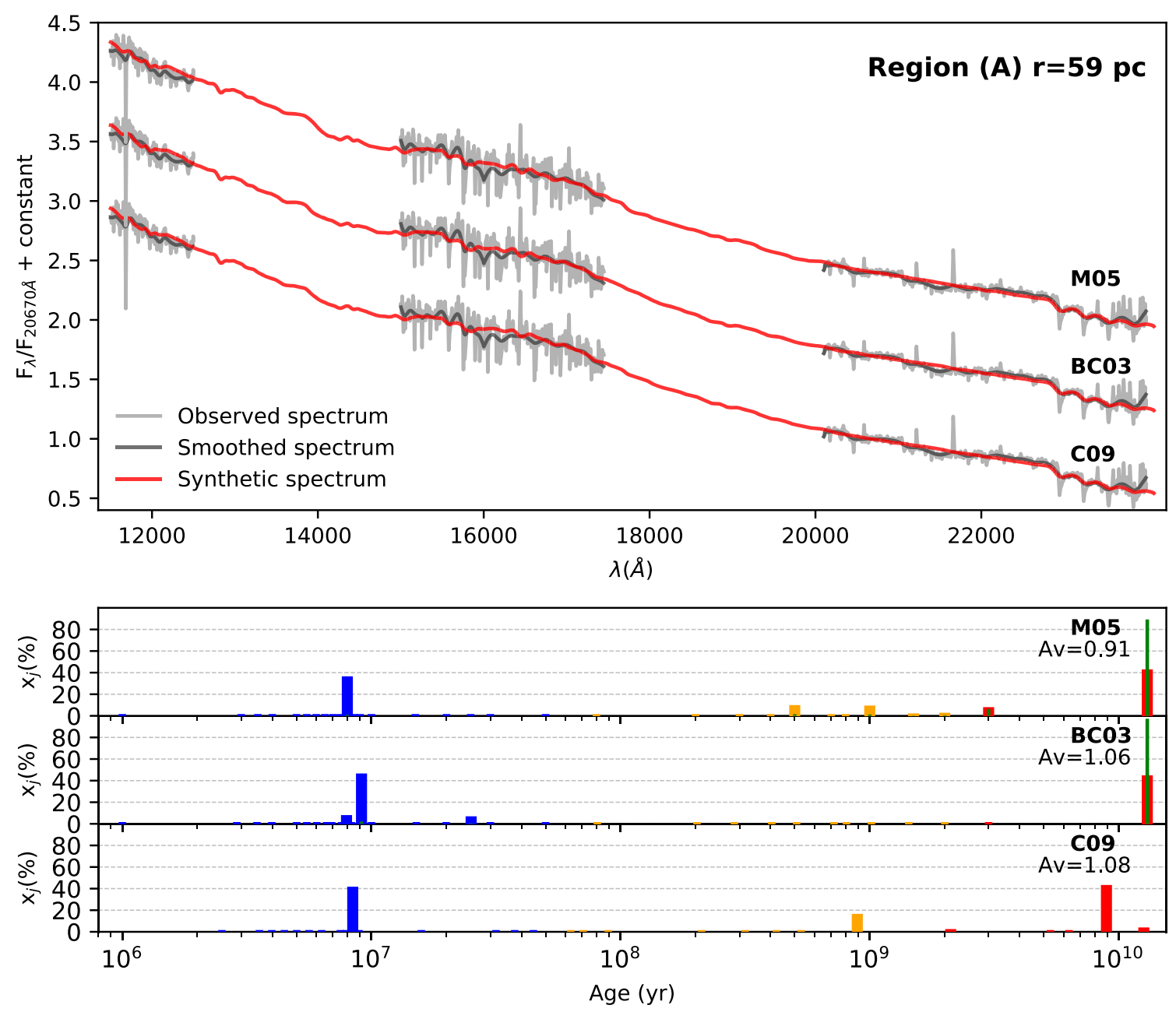

Figure 5. Same as Fig. 2, but for Region A (see Fig. 1). Results using different EPS models are presented.

tral range is less sensitive to reddening variations when using full spectrum fitting (Baldwin et al. 2018; Dahmer-Hahn et al. 2018).

In Figs 6 and 7 we present the spatial distribution of the SPCs using BC03 and C09 models. The same trend of age stratification found using M05 models is reproduced in these figures: The young SPC is distributed along the circumnuclear region with clear knots shifted eastwards from the photometric center and the spiral-arm structure found in the UV (blue contours); the old SPC is distributed outside these knots, with a clear increase in the contribution towards northwest; the intermediate-age contribution is almost negligible using these sets of models, with small knots in the south region for $\mathrm{BC} 03$ and next to the centre (co-spatial with the intermediate-age blob found using M05 models) for C09.

In order to better compare the results, we present maps with the spatially resolved differences in the SPC vectors (flux-weighted) between the three models in Fig. 8. From the two first columns, we can see BC03 and C09 display higher contribution of the $x_{y}$ SPC (redder colours in the left-hand panels), while M05 favours the $x_{i}$ and $x_{o}$ SPCs (bluer colours in the middle and right panels). Major differences are found in the southern region, co-spatial with the residual 'instrumental fingerprint' from SINFONI data (see Section 2.2), which may be the cause of these discrepancies in this region. Moreover, the intermediate-age blob found using M05 models appears to be spread in the older ages using BC03 and $\mathrm{C} 09$ models, while the inner spiral-arm clearly seen in the mass-weighted M05 maps (Fig. 3) and traced by bluer regions in the middle panels of Fig. 8 is missed using BC03 and C09 models.

The smallest differences are displayed between BC03 and C09 models, as can be seen from the bottom column in Fig 8. These two models also present nearly negligible contribution of the $x_{i}$ SPC, in contrast to results produced using M05 models. This could be related to the different prescription plus treatment of the TP-AGB phase for the different models. For example, Baldwin et al. (2018) argue their results favour Maraston \& Strömbäck (2011) treatment of the TP-AGB phase (same used in M05), in agreement with Riffel et al. (2015). Baldwin et al. (2018) also claim that the discrepancies found by Zibetti et al. (2013), which states M05 models overestimate the TP-AGB contribution, are probably related to the technique used in Zibetti et al. (2013), consisting of measuring line indices rather than full-spectrum fitting.

We decided to discuss only the results for M05 in Section 5, since these models present a proper treatment of the TP-AGB phase as discussed above, as well as the information needed to calculate the percentage mass contributions of the stellar populations. Moreover, this will allow us to compare the results presented here with those previously published by our group using M05 models (e.g. Riffel et al. 2010, 2011c; Storchi-Bergmann et al. 2012; Dametto et al. 2014; Diniz et al. 2017; Schönell et al. 2017). 

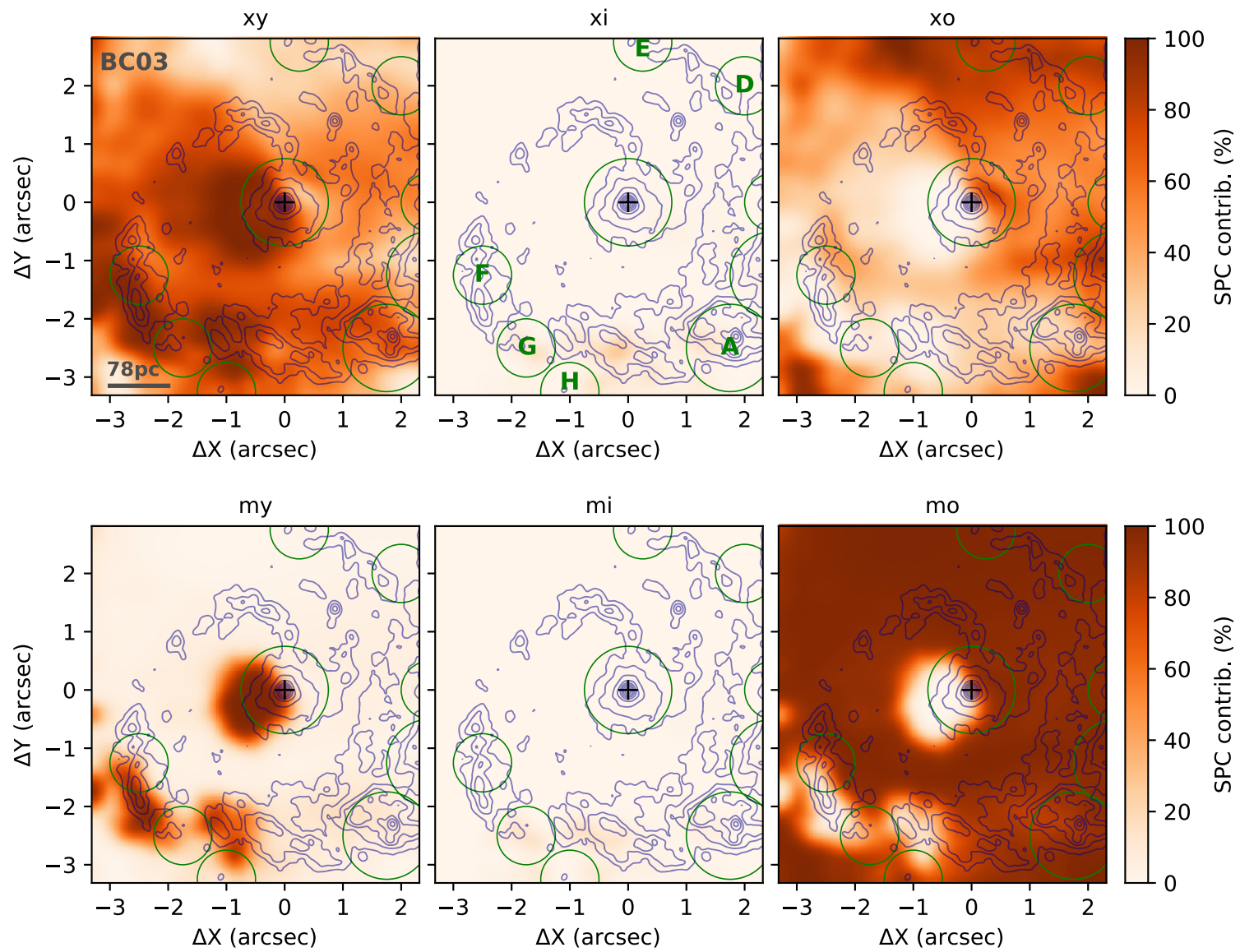

Figure 6. Same as Fig. 3, but using BC03 models.
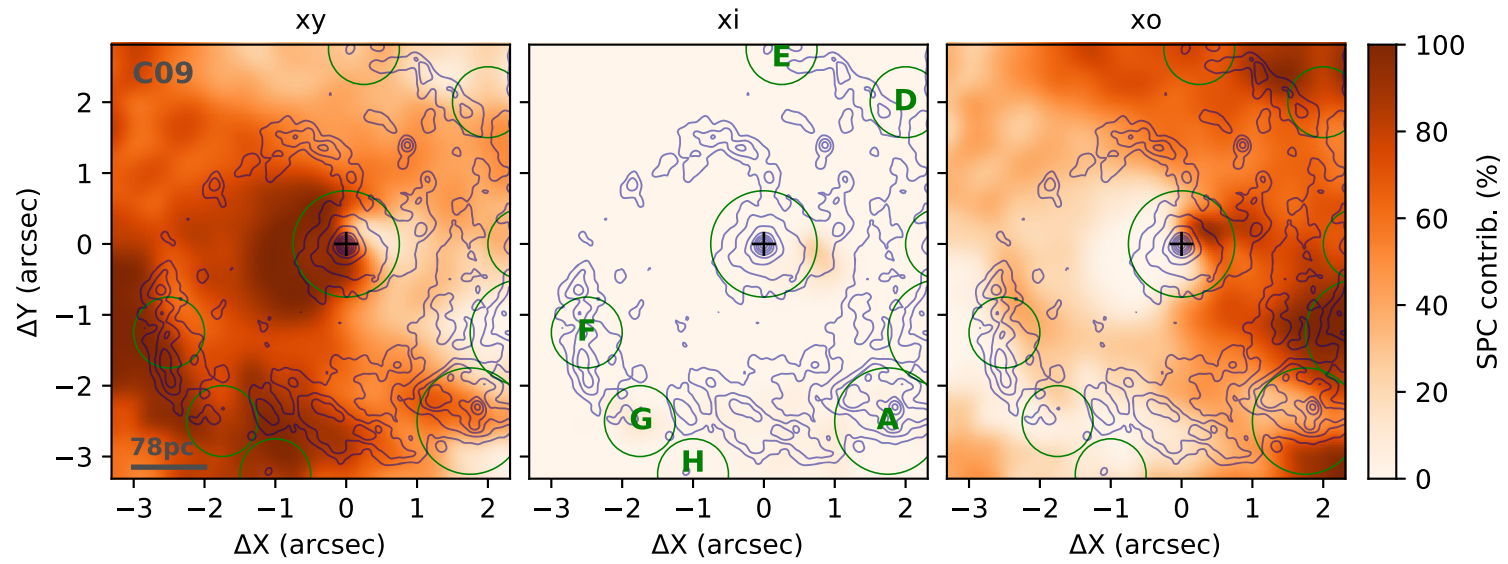

Figure 7. Same as Fig. 3, but using C09 models. As these models do not provide the mass-to-light ratio needed to calculate the mass-weighted contributions, we only present the maps for the flux-weighted contribution of each SPC.

\section{DISCUSSION}

\subsection{Distribution of the stellar populations: circumnuclear ring and spiral structures}

Stellar population distribution in the inner $\sim 200 \mathrm{pc}$ radius of NGC 4303 shows the presence of young and intermediate-age stars in a circumnuclear ring structure (see the mean age maps presented in Fig. 9), while the older components are distributed mainly along the more external parts of our FoV and we interpret them as the un- derlying bulge component. Previous 2D mapping studies of our AGNIFS group on Seyfert galaxies showed a predominance of intermediate-age stars in the central kiloparsecs of these objects (Riffel et al. 2010, 2011c; Storchi-Bergmann et al. 2012; Schönell et al. 2017), while our results point to a more conspicuous contribution of this SPC in the LLAGN NGC 4303: a blob southwest from the centre that extends to an internal spiral-arm like structure surrounding the central blob of young stars. These features suggest that star formation is stratified along the inner regions of NGC 4303, with 

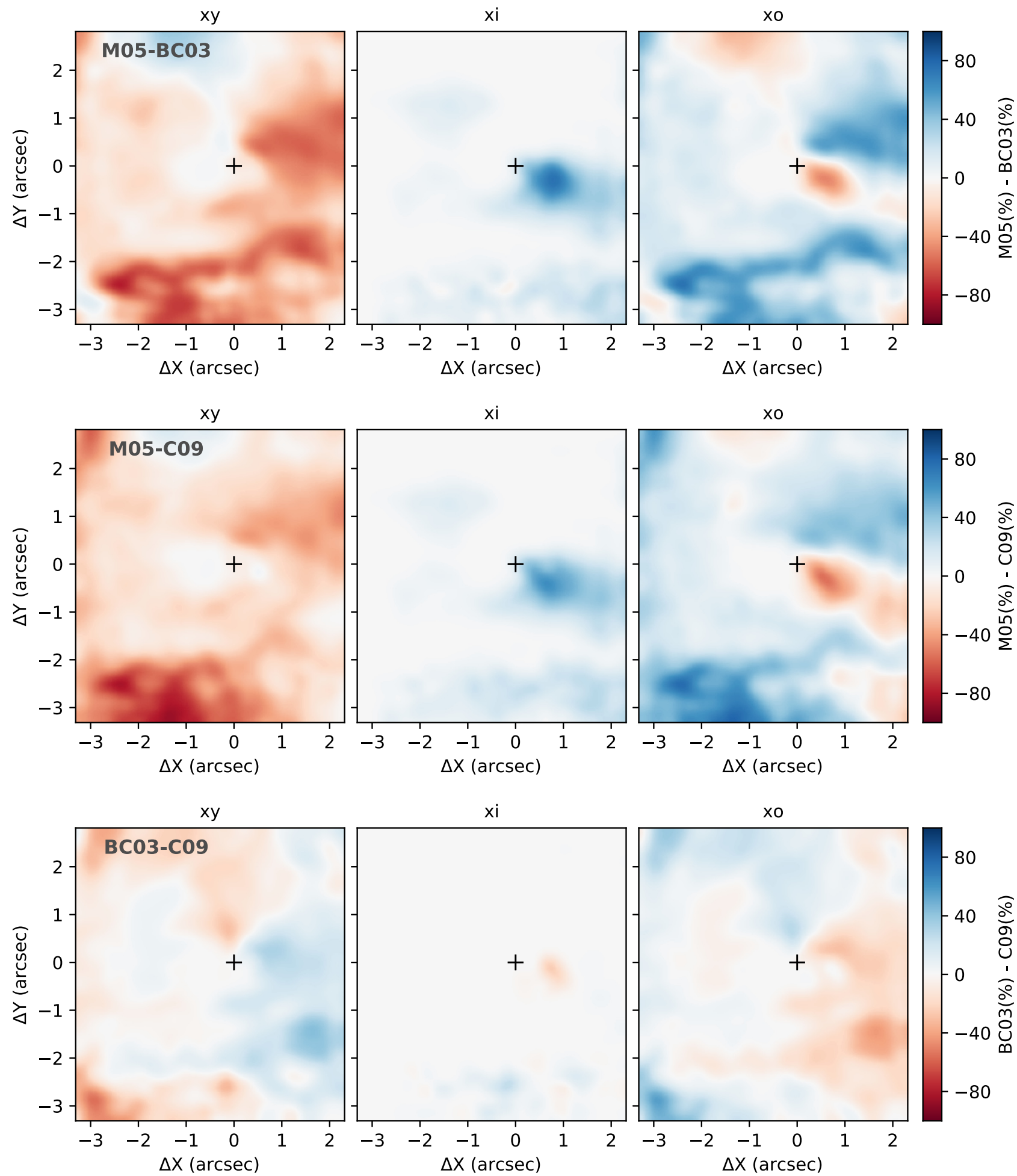

Figure 8. Comparison between the results produced with the different EPS models. Maps displaying the difference between the flux-weighted SPCs presented in Figs 3, 6, and 7.

older knots shifted towards west of the centre, while the younger ones are to the east. $\mathrm{C} 02$ and R16 reported younger ages for the western knots, which are missed by our smaller FoV, it is important to highlight.

As discussed in R16, our results favour the interpretation of a circumnuclear ring with young/intermediate-age stars rather than a spiral-arm structure proposed by $\mathrm{C} 02$. We suggest the spiral structure traced by the UV emission is in fact tracing a region of low extinction inside the disc containing the circumnuclear ring. This interpretation is in agreement with previous results from $\mathrm{C} 00$. These authors presented a $V-H$ image of the central region of this source (see their fig. 3), which reveled a rather complex gas/dust and 

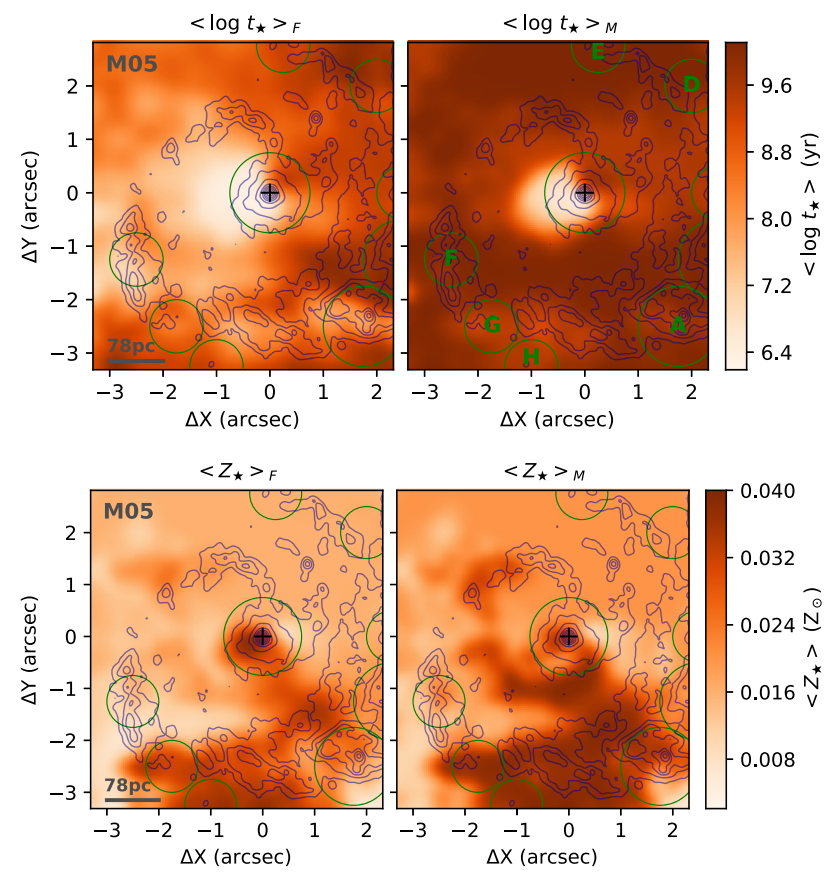

Figure 9. Logarithm of the mean age (top panels) and mean metallicity (bottom panels) weighted by flux (left) and weighted by mass (right). Black cross represents the centre, blue contours from UV image and green circles marking the CNSFRs (see Fig. 1 for more details).

stellar distribution with two-arm (star forming lane in the southwest and west regions, and dust lane at northeast) spiral structure.

In the aim of better visualizing these findings, we constructed mean value maps. A more compressed but also useful way to represent the stellar population mixture in the galaxy is by computing the mean stellar age (flux and mass-weighted, respectively), as defined by Cid Fernandes et al. (2005a):

$\left\langle\log t_{\star}\right\rangle_{F}=\sum_{j=1}^{N_{\star}} x_{j} \log \left(t_{j}\right)$,

$\left\langle\log t_{\star}\right\rangle_{M}=\sum_{j=1}^{N_{\star}} m_{j} \log \left(t_{j}\right)$.

and mean stellar metallicity:

$$
\begin{aligned}
\left\langle Z_{\star}\right\rangle_{F} & =\sum_{j=1}^{N^{\star}} x_{j} Z_{j}, \\
\left\langle Z_{\star}\right\rangle_{M} & =\sum_{j=1}^{N^{\star}} m_{j} Z_{j} .
\end{aligned}
$$

We present the maps for these parameters in Fig. 9. From the mean age maps we can see the youngest stellar populations are co-spatial with the UV knots traced by the blue contours as well as the dustier regions at north-east probed by optical imaging, as mentioned before. A comparison between the mean age and mean metallicity maps suggests the presence of a rather young metalrich $\mathrm{SPC}\left(\sim 2 \mathrm{Z}_{\odot}\right)$ in the nuclear region of NGC 4303, as well as in the southern region, where the youngest CNSFRs encompassed by our FoV are located. Moreover, higher metallicity values (up to $2 \mathrm{Z} \odot$ ) are tracing the inner spiral-arm structure of intermediate-age

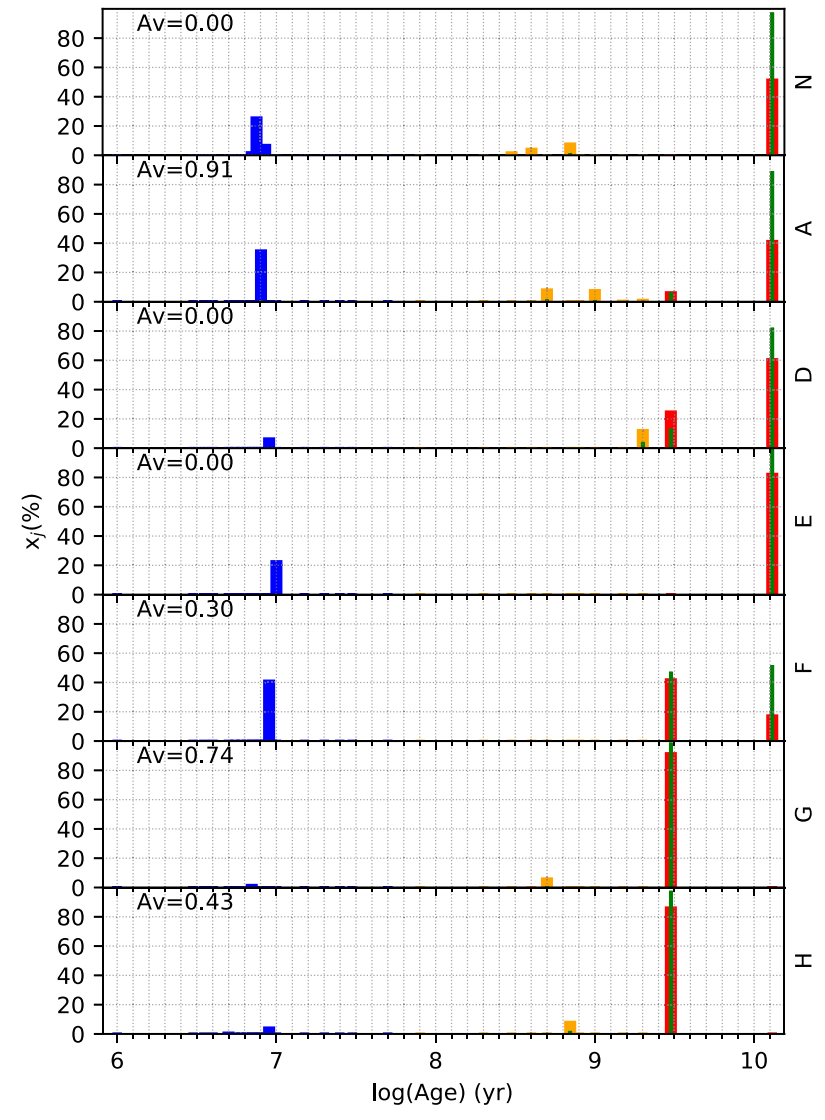

Figure 10. SP synthesis results for the CNSFRs in NGC 4303. Each panel displays the percent contribution in flux (colour bars) and mass (green lines) at $\lambda=2.067 \mu \mathrm{m}$ of the stellar population vectors in each region. Blue, orange, and red represent young $\left(x_{y}\right)$, intermediate-age $\left(x_{i}\right)$, and old $\left(x_{o}\right)$ SPCs contributions, respectively. The letters correspond to the regions identified by R16 and marked as green circles in our maps.

stars (see also Fig. 3). These results give further support to the age stratification scenario proposed for the $\mathrm{SFH}$ in this source.

\subsection{Stellar populations in the CNSFRs}

In R16 we present new maps of emission-line flux distributions and kinematics in both ionized and molecular gas in the inner $350 \mathrm{pc}$ radius (a slightly wider FoV than the one we use in this work; see Section 4) of NGC 4303. The most prominent feature is a 200 $250 \mathrm{pc}$ ring of CNSFRs, which is seen as a nuclear spiral in UV/HST images presented in $\mathrm{C} 02$. In order to better analyze and compare our results with those obtained from the study of the emission-lines in R16, we decided to extract the spectra of the CNSFRs encompassed by our FoV, matching the aperture defined in that work. The spectra were extracted as the summed flux inside each aperture (Regions: $\mathrm{N} / \mathrm{A}, r=60 \mathrm{pc}$; D/E/F/G/H, $r=39 \mathrm{pc}$ ).

In Fig. 10 we display a series of histograms with the stellar population synthesis results (also summarized in Table 1) for the CNSFRs: Each panel represents the results for the respective region marked in green circles in our maps. From these plots it is clear the presence of young stars (7-10 Myr) coexisting with an older SPC, which we attribute to the underlying bulge component (3/13 Gyr). A small contribution of up to 20 per cent of intermediate-age stars with a large spread in age $(0.3-2.0 \mathrm{Gyr})$ is seen in the CNSFRs, with exception of apertures $E$ and $F$. Our results point out to a 
Table 1. Synthesis results for the CNSFRs shown in Fig. 10 (green circles in the maps).

\begin{tabular}{|c|c|c|c|c|c|c|c|c|c|c|c|c|c|c|}
\hline Region & $\begin{array}{c}x_{y} \\
(\%) \\
(1)\end{array}$ & $\begin{array}{c}x_{i} \\
(\%) \\
(2)\end{array}$ & $\begin{array}{l}x_{o} \\
(\%) \\
(3)\end{array}$ & $\begin{array}{l}m_{y} \\
(\%) \\
(4)\end{array}$ & $\begin{array}{c}m_{i} \\
(\%) \\
(5)\end{array}$ & $\begin{array}{l}m_{o} \\
(\%) \\
(6)\end{array}$ & $\begin{array}{c}\left\langle\log t_{\star}\right\rangle_{F} \\
(\mathrm{yr}) \\
(7)\end{array}$ & $\begin{array}{c}\left\langle\log t_{\star}\right\rangle_{M} \\
(\mathrm{yr}) \\
(8)\end{array}$ & $\begin{array}{c}\mathrm{Z}_{F} \\
\left(\mathrm{Z}_{\odot}\right) \\
(9)\end{array}$ & $\begin{array}{c}\mathrm{Z}_{M} \\
\left(\mathrm{Z}_{\odot}\right) \\
(10)\end{array}$ & $\begin{array}{c}\mathrm{A}_{V} \\
(\mathrm{mag}) \\
(11)\end{array}$ & $\begin{array}{c}\mathrm{SFR} \\
\left(\mathrm{M}_{\odot} \mathrm{yr}^{-1}\right) \\
(12)\end{array}$ & 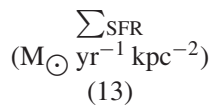 & $\begin{array}{l}\text { Adev } \\
(\%) \\
(14)\end{array}$ \\
\hline$(\mathrm{N})$ & 35 & 14 & 51 & 0 & 2 & 97 & $6.17 \times 10^{8}$ & $1.74 \times 10^{10}$ & 1.81 & 1.98 & 0 & 0.426 & 39.62 & 1.04 \\
\hline (A) & 35 & 18 & 47 & 1 & 4 & 96 & $5.50 \times 10^{8}$ & $1.00 \times 10^{10}$ & 1.20 & 1.83 & 0.91 & 0.144 & 13.39 & 1.65 \\
\hline (D) & 6 & 12 & 82 & 0 & 4 & 96 & $4.68 \times 10^{9}$ & $9.77 \times 10^{9}$ & 1.00 & 1.00 & 0 & 0.005 & 1.0 & 2.90 \\
\hline (E) & 22 & 0 & 78 & 0 & 0 & 100 & $2.75 \times 10^{9}$ & $1.29 \times 10^{9}$ & 1.00 & 1.00 & 0 & 0.018 & 3.66 & 3.35 \\
\hline (F) & 41 & 0 & 59 & 1 & 0 & 99 & $3.63 \times 10^{8}$ & $6.17 \times 10^{9}$ & 1.33 & 1.21 & 0.30 & 0.046 & 9.71 & 1.98 \\
\hline
\end{tabular}

Note. (1), (2), (3): average contribution in flux of the young (y: $\leq 50 \mathrm{Myr})$, intermediate-age (i: 0.05-2 Gyr), and old (o: > 2 Gyr) SP component, respectively; (4), (5), (6): average contribution of the SP components in mass; (7), (8): flux- and mass-weighted mean ages; (9), (10): flux- and mass-weighted mean metallicities; (11): visual extinction; (12): SFR; (13): star formation surface density; and (14): per cent mean deviation.

more complex nuclear ring SFH, with multiple starburst episodes, in agreement with recent results (Ma, de Grijs \& Ho 2018).

Previous studies have found an age dating for the star clusters distributed along the circumnuclear ring-like structure in NGC 4303. C00 found the east UV knots (blue contours in our maps) are older ( $\sim 10-25 \mathrm{Myr})$ than the west knots $(\sim 5-7.5 \mathrm{Myr})$, thus suggesting an age offset in the ring.

Even though emission-line measurements done by R16 show a trend suggesting an age sequence along the ring increasing from $A$ to $H$ (see figs 4 and 9 from R16), the distribution of CNSFRs and their age differences indicate a rather episodic star formation along the ring, being equally consistent with regions to the east being older than regions to the west, in agreement with $\mathrm{C} 00$. For example, from $\mathrm{H}_{2} / \mathrm{Br} \gamma$ ratios, they found regions $G$ and $H$ to be older than regions $A-F$, in full agreement with our results, as can be seen in Fig. 10 .

In Table 1 (column 12), we present the SFRs derived for all the apertures. We can see Region $A$ displays the highest SFR $\left(0.14 \mathrm{M}_{\odot} \mathrm{yr}^{-1}\right)$ in the ring, co-spatial with high $\mathrm{H} \alpha$ emission as presented by Colina \& Arribas (1999). The range of the SFRs in the circumnuclear ring goes from $0.002-0.14 \mathrm{M}_{\odot} \mathrm{yr}^{-1}$, displaying $\sim 1$ order of magnitude higher than those presented in R16 using the $\mathrm{Br} \gamma$ luminosity and assuming a constant rate. These discrepancies might be related to the fact that with the stellar population fitting we are probing different (older) starbursts than those which are effectively ionizing the gas. In fact, Ma et al. (2018) found evidence suggesting that the SFH in nuclear rings are better described by models of multiple bursts of star- formation, rather than assuming a constant SFR. In this work, the authors derived the age of the circumnuclear ring of NGC 4303 through SED fitting and found it to be around $3 \mathrm{Gyr}$ (using $\mathrm{C} 09$ and assuming $Z=\mathrm{Z}_{\odot}$ ), in agreement with our results (see column 7 of Table 1).

In order to better compare our results with the literature, we divided the SFR by the area of the extractions, obtaining the star formation surface density ( $\sum_{\mathrm{SFR}}$, see column 13 of Table 1$)$. In the nucleus, $\sum_{\mathrm{SFR}}$ reaches up to $\sim 40 \mathrm{M}_{\odot} \mathrm{yr}^{-1} \mathrm{kpc}^{-2}$, while in the ring values are in the range of $0.5-13 \mathrm{M}_{\odot} \mathrm{yr}^{-1} \mathrm{kpc}^{-2}$. Typical star formation surface density values range from $1-50 \mathrm{M}_{\odot} \mathrm{yr}^{-1} \mathrm{kpc}^{-2}$ on hundreds of parsec scales, $50-500 \mathrm{M}_{\odot} \mathrm{yr}^{-1} \mathrm{kpc}^{-2}$ on scales of tens of parsecs, while it increases up to $\sim 1000 \mathrm{M}_{\odot} \mathrm{yr}^{-1} \mathrm{kpc}^{-2}$ on parsec scales (Valencia-S. et al. 2012, and references therein). Our $\sum_{\mathrm{SFR}}$ values for the CNSFRs, including the nucleus (inner $\sim 120 \mathrm{pc}$ ) are within the first $\sum_{\mathrm{SFR}}$ ranges. This is in agreement with previous results, such as those presented by Busch et al. (2017), which found $\sum_{\mathrm{SFR}}$ of $\sim 28 \mathrm{M}_{\odot} \mathrm{yr}^{-1} \mathrm{kpc}^{-2}$ in the nucleus and $5-13 \mathrm{M}_{\odot} \mathrm{yr}^{-1} \mathrm{kpc}^{-2}$ in the ring of the nearby barred galaxy
NGC 1808. Thus, confirming are results display typical star formation surface density values.

Based on the spatial distributions of CNSFRs in nuclear rings, for example, Böker et al. (2008) discussed two scenarios of star formation: the 'pop-corn' scenario in which the stellar clusters form at random positions producing no systematic age gradients and, the 'pearls-on-a-string' scenario in which the clusters are formed where the gas enters the ring and then age as they orbit the ring forming a string of aging clusters (see also Ryder, Knapen \& Takamiya 2001; Díaz-Santos et al. 2007). R16 could not favour either of the two scenarios from the emission-line study, suggesting that stars formed quasi-simultaneously over a large sector of the ring, aging as they rotate with an orbital time of $\sim 10 \mathrm{Myr}$. Seo \& Kim (2013) performed simulations on how star formation proceed in nuclear rings of barred galaxies and found a critical SFR $\left(\mathrm{SFR}_{\mathrm{c}}\right)$ of $\sim 1 \mathrm{M}_{\odot} \mathrm{yr}^{-1}$, which determines how star formation takes place in these rings. When the SFR in the ring is low $\left(<\mathrm{SFR}_{\mathrm{c}}\right)$, star formation mostly takes place in the contact points of the ring with dust lanes leading to an age gradient ('pearls-on-a-string'). Otherwise, if the SFR is high $\left(>\mathrm{SFR}_{c}\right)$, then star formation is randomly distributed along the ring ('pop-corn'). Mazzuca et al. (2008) found similar results studying 22 nuclear rings (with SFRs in the range of 01$10 \mathrm{M}_{\odot} \mathrm{yr}^{-1}$ ), but for a higher $\mathrm{SFR}_{\mathrm{c}}$ of $\sim 3 \mathrm{M}_{\odot} \mathrm{yr}^{-1}$. Summing up the SFRs of the CNSFRs (excluding the nuclear aperture), we ended up with an SFR $\sim 0.2 \mathrm{M}_{\odot} \mathrm{yr}^{-1}$, a typical value for nuclear rings (Ma et al. 2018). Even if we add the mean SFR of the northeast section of the ring $\left(\leq 2.5 \times 10^{-3} \mathrm{M}_{\odot} \mathrm{yr}^{-1}\right.$; see Fig. 4), the SFR in the circumnuclear ring of NGC 4303 would still be lower than the $\mathrm{SFR}_{\mathrm{c}}$ proposed by these previous results. Thus, our SFR results favour the 'pearls-on-a-string' scenario, with a gradient of increasing age going from regions $A$ to $H$.

When comparing the reddening values derived from the emission lines in R16 with those found with our stellar population fitting, the former present higher $\mathrm{A}_{V}$ (reaching up to $2.5 \mathrm{mag}$ in Regions $B$ and $E)$. This is expected and is related to the fact that the hot ionizing stars are associated to dustier regions with respect to the cold stellar population (Calzetti, Kinney \& Storchi-Bergmann 1994). The ring displays reddening values from 0 to $0.9 \mathrm{mag}$, lower than typical values from previous studies ranging from 2 to 5 mag (Krabbe, Sternberg \& Genzel 1994; Kotilainen et al. 1996; Rosenberg, van der Werf \& Israel 2012; Busch et al. 2017), but consistent with UV and optical results from $\mathrm{C} 00$ for less obscured regions in the $\mathrm{V}-\mathrm{H}$ colours, as well as with Cluster $\mathrm{G}$ of $\mathrm{C} 02$ with $\mathrm{A}_{V}=0.6 \mathrm{mag}$ (using $\left.R_{V}=4.05\right)$, which we call Region $A$.

In addition, the Adev values in Fig. 10, column (12) illustrate the good quality of the fits for the CNSFRs. 


\subsection{Stellar populations in the nuclear region: is there any evidence of the LLAGN?}

The presence of an AGN in the central region of NGC 4303 is still a matter of debate. Using NIR emission-line ratios, R16 constructed a NIR diagnostic diagram (proposed by Colina et al. 2015), which suggested the presence of an AGN at the nucleus, confirming previous results that the nuclear emission of this galaxy has a composite nature (LLAGN plus a young massive stellar cluster; e.g. Colina et al. 2002; Jiménez-Bailón et al. 2003).

As already mentioned in Section 1, previous studies suggested a young (4 Myr) massive SSC dominates the UV emission, while an intermediate/old (1-5Gyr) stellar population dominates the optical continuum $(\mathrm{C} 00, \mathrm{C} 02)$. Our results in the nuclear region (inner $60 \mathrm{pc}$ radius) of this source show the same trend, presenting a contribution of young stars with rather older ages (7-8.5 Myr) and a rather younger intermediate-age SPC $(0.3-0.7$ Gyr $)$ plus a dominating ( $\sim 50$ per cent) 13 Gyr old stellar population, which we attribute to the underlying bulge. Moreover, $\mathrm{C} 00$ suggested that the $H$ excess in the inner $8 \mathrm{pc}$ could be related to the presence of luminous red supergiants, implying a second star formation episode with $\sim 10 \mathrm{Myr}$, which are close in age with the young stars we found. These authors also argued the large $V-H$ colours (3.2 mag) found in this inner region could not be fully accounted for by small amounts of dust extinction and suggested the presence of an extremely red source, such as a hidden AGN. Assuming a pure power law with $v=-1$, representing the AGN featureless emission, they obtained values of $V-H=2.3 \mathrm{mag}$, closer to the observed ones, but still not fully compatible.

From the stellar population synthesis we do not find contribution of the featureless continuum and/or hot emission component to the inner $60 \mathrm{pc}$ radius. Although this result does not rule out the existence of a hidden AGN in the centre of NGC 4303, it supports the scenario of an LLAGN/LINER-like source rather than a Seyfert 2 nucleus (Colina \& Arribas 1999). In a recent work, Burtscher et al. (2015) found no evidence of nuclear EW dilution of the CO feature by AGN light in this source (as can be seen in their fig 2), in agreement with our stellar population analysis. It is important to call attention to the fact that the spectra of very young SSPs $(t \leq$ $5 \mathrm{Myr}$; Riffel et al. 2009b, and references therein) and the featureless continuum emission can be degenerated in the fits, meaning we cannot fully discriminate between these two components using our methodology. Nevertheless, we are favourable to believe that the young SPC we are finding with stellar population synthesis is real, since there is more than sufficient evidence in the literature of the presence of young stars in the nuclear region of this source. Furthermore, our fits find SSPs in the range of 7-8.5 Myr, which are not the younger SSPs $(t \lesssim 5 \mathrm{Myr})$ that usually mimic the contribution of a featureless continuum (Cid Fernandes et al. 2004).

Additional support for the scenario of an LLAGN/LINER-like source rather than a Seyfert 2 nucleus comes from the fact that the inclusion of a featureless continuum (power law) and hot dust emission (blackbody functions) has been used to trace signatures of luminous AGNs by our AGNIFS group when performing stellar population synthesis in the NIR, and in the case of Seyfert sources these components are required to properly fit their spectral energy distribution.

\subsubsection{Nuclear extinction}

Typical nuclear reddening values $\left(\mathrm{A}_{V}\right)$ for Seyfert galaxies derived using NIR emission-lines are in the range of 1.3-5 mag for Type 1 nuclei and 1.8-9.0 mag for Type 2 (Valencia-S. et al. 2012, and references therein). These authors also derived a 2.5 mag reddening for the nuclear region of a starburst/Seyfert composite galaxy. In the same line, Dametto et al. (2014) found nuclear $\mathrm{A}_{V}$ ranging from 2.5 to 8.0 mag for the gas in four Starburst galaxies, while for the stars they found 1.0-3.2 mag. In addition, AGNIFS group derived nuclear reddening values using NIR data from Seyfert 2 galaxies and found values between 2 and 4 mag for the stars, while for the gas these values reach up to 7.0 mag in Mrk 573 (Riffel et al. 2010, 2011c; Diniz et al. 2017).

Previous reddening estimations were made for NGC 4303 in C02 using the emission lines as well as the UV shape of the spectrum for the nuclear region. These authors found $\mathrm{A}_{V}=0.2 / 0.4$ mag using UV spectra (LMC/Calzetti extinction law), while using $\mathrm{H} \alpha / \mathrm{H} \beta$ line ratio they fitted a dust-free nuclear spectrum $(\mathrm{r}=0$ ".45-35 $\mathrm{pc})$ for this object. These low extinction values are in agreement with our results for the nuclear extraction $(\mathrm{r}=0$.'75 $60 \mathrm{pc}$ ), as can be seen in the top panel of Fig. 10. However, when looking to the results spaxel-by-spaxel in Fig. 4 (left-hand panel), we derived rather higher $A_{V}$ values ( $\sim 2.3 \mathrm{mag}$ ) for the inner tens of parsecs in this source, which is within the typical reddening values. $\mathrm{C} 00$ had found large $V-H$ colours (3.2 mag) for the inner regions ( $<8 \mathrm{pc}$ ) of NGC 4303, as already mentioned, arguing that this could possibly be related to a hidden AGN, which is consistent with our results (see Section 5.3).

\subsubsection{Nuclear SFRs}

In Table 1 we present SFR values for the nuclear region $\left(0.43 \mathrm{M}_{\odot} \mathrm{yr}^{-1}\right)$, which are in the range of typical values found in the literature (see also Section 5.2). For example, using $\mathrm{Br} \gamma$ luminosity, Valencia-S. et al. (2012) and Busch et al. (2017) derived nuclear SFRs of $0.18 \mathrm{M}_{\odot} \mathrm{yr}^{-1}$ and $<0.35 \mathrm{M}_{\odot} \mathrm{yr}^{-1}$, respectively, in the inner $57 / 50 \mathrm{pc}$ radius in their sample. Jiménez-Bailón et al. (2003) and R16 also calculated the SFR in the inner hundreds of parsecs in NGC 4303 using emission line indicators in the optical and NIR, respectively, and both found $0.013 \mathrm{M}_{\odot} \mathrm{yr}^{-1}$, a lower value than the one we are finding. As discussed in Section 5.2, this might be related to the different ages of the bursts we are probing with stellar populations synthesis, which considers a wide range of bursts up to 10 Myr. When using emission line indicators to derive SFRs, one is looking into a more instantaneous burst scenario, favouring the youngest components of the stellar population.

Assuming the SFR of $0.43 \mathrm{M}_{\odot} \mathrm{yr}^{-1}$ for the last $10 \mathrm{Myr}$, the inner $60 \mathrm{pc}$ radius of this source would have formed $\sim 4 \times 10^{6} \mathrm{M}_{\odot}$. Then, if we assume an efficiency of $\sim 0.1$ to convert gas into stars, this region should comprise around $10^{7} \mathrm{M}_{\odot}$ of cold molecular $\left(\mathrm{H}_{2}\right)$ gas $\left(M_{\text {cold }}\right)$, which is in agreement with the values found by previous studies for the cold molecular gas in this region of NGC 4303 , such as Schinnerer et al. (2002) and R16. The former derived $M_{\text {cold }}=6.9 \times 10^{7} \mathrm{M}_{\odot}$ directly from CO observations of the nuclear disc of this object, while the later estimated $M_{\text {cold }}=6.25 \times 10^{6} \mathrm{M}_{\odot}$ from converting hot to cold molecular gas masses (e.g. Mazzalay et al. 2013).

An alternative way to approach this would be to compare rates of star formation, mass inflow, and mass accretion to the black hole. Mass-inflow rates are in the range of $10^{-2}-10 \mathrm{M}_{\odot} \mathrm{yr}^{-1}$, which is consistent with our nuclear SFR $\left(0.43 \mathrm{M}_{\odot} \mathrm{yr}^{-1}\right)$ plus a typical mass accretion rate to the black hole of $10^{-3}-10^{-2} \mathrm{M}_{\odot} \mathrm{yr}^{-1}$ (Riffel 2013, and references therein). Thus, the values we are finding for 
the SFR in the central region of this source are feasible with the amount of gas available to form stars.

\section{CONCLUSIONS}

We present the first spatially resolved stellar population study of the inner $\sim 200 \mathrm{pc}$ radius of NGC 4303 in the NIR. Using $J_{-}, \mathrm{H}$-, and $K$-band SINFONI/VLT data, stellar population synthesis was performed with the STARLIGHT code and M05 SSP models. The main conclusions of this work go as follows:

(i) The dominant stellar population component presents a spatial variation in the inner $\sim 200 \mathrm{pc}$ radius of this source, suggesting an age stratification. The youngest stellar population components ( $t \leq 2 \mathrm{Gyr}$ ) are distributed along a circumnuclear ring with 200$250 \mathrm{pc}$ radius in agreement with previous studies (R16). Three main components stand out: two nuclear blobs, one composed by young stars $(t \leq 50 \mathrm{Myr})$ and shifted towards east from the centre (here defined as the peak of the $\mathrm{Br} \gamma$ emission line) and one composed by intermediate-age stars (50 Myr $<t \leq 2 \mathrm{Gyr}$ ) located southwest from the centre; and an internal spiral arm-like structure also composed by intermediate-age stars surrounding the blob of young stars. The old stellar population component that we attribute to an underlying bulge stellar population is distributed outside the two blob structures, with an enhanced contribution northwest from the centre. These results reveal a rather complex SFH in NGC 4303, indicating that star formation has occurred through multiple bursts in this source.

(ii) With our stellar population synthesis analysis, we favour the interpretation of a circumnuclear ring of star formation in the inner $\sim 250$ pc radius of NGC 4303 rather than a spiral arm structure as suggested by $\mathrm{C} 02$, in agreement with the analysis of the emission gas presented in R16. We suggest the spiral arm seen in UV images is tracing the less obscured star-forming regions of the circumnuclear ring.

(iii) Circumnuclear star-forming regions (CNSFRs) distributed along the ring present SFRs in the range of $0.002-0.14 \mathrm{M}_{\odot} \mathrm{yr}^{-1}$. Our results favour the 'pearls-on-a-string' star formation scenario with an age gradient along the ring. This conclusion is based on the fact that we have found a value for the total SFR in the ring that is lower than the critical SFR $\left(\sim 1 \mathrm{M}_{\odot} \mathrm{yr}^{-1}\right)$ to separate between the 'pearls-on-a-string' and the 'pop-corn' scenarios.

(iv) At the nuclear region $(R \lesssim 60 \mathrm{pc})$ we find a series of star formation bursts: a first one occurring 13 Gyrs ago $\left(x_{o}\right)$, accounting for 50 per cent of the light at $2.067 \mu \mathrm{m}$; a set of individual minor bursts with ages from 0.3 to $0.7 \mathrm{Gyr}\left(x_{i}\right)$ corresponding to $\sim 15$ per cent of the light and a contribution of $\sim 35$ per cent of the $x_{y}$ component with a major burst ( $\sim 30$ per cent) at $7.5 \mathrm{Myr}$. We derive a nuclear SFR of $0.43 \mathrm{M}_{\odot} \mathrm{yr}^{-1}$, corresponding to a star formation surface density $\left(\sum_{\mathrm{SFR}}\right)$ of $\sim 40 \mathrm{M}_{\odot} \mathrm{yr}^{-1} \mathrm{kpc}^{-2}$.

(v) No signatures of non-thermal featureless continuum and hot dust (blackbody functions) components were necessary to reproduce the nuclear (inner $60 \mathrm{pc}$ ) continuum of this source. This result supports the scenario in which an LLAGN/LINER-like source is hidden in the centre of NGC 4303, rather than a Seyfert 2 nucleus. In the case of Seyferts, such components are required to properly reproduce their spectral energy distribution.

(vi) A reddening free nuclear spectrum $(R \lesssim 60 \mathrm{pc})$ was fitted for this source, in agreement with UV and optical previous results. When looking to the most inner (unresolved) regions ( $<10 \mathrm{pc}$ ), we find a reddened solution consistent with the presence of a hidden
AGN, as already proposed by $\mathrm{C} 00$ from the large $V-H$ colours (3.2 mag) in this region.

(vii) From our spatially resolved comparison of the results obtained using different SSP models, we concluded the use of M05 models, in general, yields a more consistent scenario for the distribution of the stellar populations. Results using BC03 and C09 models miss the contribution of intermediate-age stars, which were expected to be found in active star formation sources. We speculate the main reason for discrepancies found between the results using these three low-resolution EPS models sets is related to their prescription for implementing the TP-AGB phase to the models.

As LLAGNs are the most common type of nuclear activity in the Local Universe, the understanding of their nature and SFH is of utmost importance for the comprehension of galaxy formation and evolution. Our results on the spatially resolved stellar populations of the few hundreds of parsecs in the nearby LLAGN NGC 4303 show a complex star formation scenario, with different stellar population components coexisting with a low efficiency accreting black hole. Thus, this detailed study helps to shed some light on the contemporary scenarios of galaxy formation and evolution.

\section{ACKNOWLEDGEMENTS}

We thank an anonymous referee for useful suggestions that helped to improve the paper. The authors would like to thank Dr. Aberto Rodríguez Ardila of the Laboratório Nacional de Astronomia, Brazil, and Dr. Lucimara Martins of Universidade Cruzeiro do Sul, Brazil, for kindly sharing with us their IRTF SpeX spectrum of NGC 4303. The Brazilian authors acknowledge support from FAPERGS (Fundação de Ampáro à pesquisa do Estado do Rio Grande do Sul) and CNPq (Conselho Nacional de Desenvolvimento Científico e Tecnológico). L.C. acknowledges support from $\mathrm{CNPq}$ special visitor fellowship PVE 313945/2013-6 under the Brazilian program Science without Borders. L.C. and S.A. are supported by grants AYA2012-32295, AYA2012- 39408, ESP2015-68964-P, and ESP2017-83197-P, and J.P. is supported by grant AYA201785170-R, all from the Ministerio de Economía y Competitividad of Spain. The STARLIGHT project is supported by the Brazilian agencies CNPq, CAPES and FAPESP (Fundação de Ampáro à pesquisa do Estado de São Paulo) and by the France-Brazil CAPES/Cofecub program.

\section{REFERENCES}

Asari N. V., Cid Fernandes R., Stasińska G., Torres-Papaqui J. P., Mateus A., Sodré L., Schoenell W., Gomes J. M., 2007, MNRAS, 381, 263

Baldwin C., McDermid R. M., Kuntschner H., Maraston C., Conroy C., 2018, MNRAS, 473, 4698

Belfiore F. et al., 2016, MNRAS, 461, 3111

Bessell M. S., Brett J. M., Wood P. R., Scholz M., 1989, A\&AS, 77, 1

Bessell M. S., Brett J. M., Scholz M., Wood P. R., 1991, A\&AS, 89, 335

Bica E., 1988, A\&A, 195, 76

Bica E., Alloin D., 1986, A\&A, 162, 21

Böker T., Falcón-Barroso J., Schinnerer E., Knapen J. H., Ryder S., 2008, AJ, 135, 479

Bolzonella M., Miralles J.-M., Pelló R., 2000, A\&A, 363, 476

Bonnet H. et al., 2004, The Messenger, 117, 17

Bruzual G., Charlot S., 2003, MNRAS, 344, 1000 (BC03)

Burtscher L. et al., 2015, A\&A, 578, A47

Busch G., Eckart A., Valencia-S. M., Fazeli N., Scharwächter J., Combes F., García-Burillo S., 2017, A\&A, 598, A55

Calzetti D., Kinney A. L., Storchi-Bergmann T., 1994, ApJ, 429, 582 
Calzetti D., Armus L., Bohlin R. C., Kinney A. L., Koornneef J., StorchiBergmann T., 2000, ApJ, 533, 682

Cassisi S., degl'Innocenti S., Salaris M., 1997a, MNRAS, 290, 515

Cassisi S., Castellani M., Castellani V., 1997b, A\&A, 317, 108

Cid Fernandes R., Gu Q., Melnick J., Terlevich E., Terlevich R., Kunth D., Rodrigues Lacerda R., Joguet B., 2004, MNRAS, 355, 273

Cid Fernandes R., González Delgado R. M., Storchi-Bergmann T., Martins L. P., Schmitt H., 2005a, MNRAS, 356, 270

Cid Fernandes R., Mateus A., Sodré L., Stasińska G., Gomes J. M., 2005b, MNRAS, 358, 363

Colina L., Arribas S., 1999, ApJ, 514, 637

,Colina L., Wada K., 2000, ApJ, 529, 845 (C00)

Colina L., García Vargas M. L., Mas-Hesse J. M., Alberdi A., Krabbe A., 1997, ApJ, 484, L41

Colina L., Gonzalez Delgado R., Mas-Hesse J. M., Leitherer C., 2002, ApJ, $579,545(\mathrm{C} 02)$

Colina L. et al., 2015, A\&A, 578, A48

Combes F., 2003, in Collin S., Combes F., Shlosman I., eds, ASP Conf. Ser. Vol. 290, Active Galactic Nuclei: From Central Engine to Host Galaxy. Astron. Soc. Pac., San Francisco, p. 411

Combes F., Prugniel P., Rampazzo R., Sulentic J. W., 1994, A\&A, 281, 725

Conroy C., 2013, ARA\&A, 51, 393

Conroy C., Gunn J. E., White M., 2009, ApJ, 699, 486 (C09)

Conroy C., Villaume A., van Dokkum P. G., Lind K., 2018, ApJ, 854, 139

Dahmer-Hahn L. G., Riffel R., Rodríguez-Ardila A., Martins L. P., Kehrig C., Heckman T. M., Pastoriza M. G., Dametto N. Z., 2018, MNRAS, 476, 4459

Dametto N. Z., Riffel R., Pastoriza M. G., Rodríguez-Ardila A., HernandezJimenez J. A., Carvalho E. A., 2014, MNRAS, 443, 1754

Davies R. I. et al., 2006, ApJ, 646, 754

Davies R. I., Mueller Sánchez F., Genzel R., Tacconi L. J., Hicks E. K. S., Friedrich S., Sternberg A., 2007, ApJ, 671, 1388

de Vaucouleurs G., de Vaucouleurs A., Corwin H. G., Jr., Buta R. J., Paturel G., Fouque P., 1991, Third Reference Catalogue of Bright Galaxies, Springer, New York, NY.

Díaz-Santos T., Alonso-Herrero A., Colina L., Ryder S. D., Knapen J. H., 2007, ApJ, 661, 149

Diniz M. R., Riffel R. A., Riffel R., Crenshaw D. M., Storchi-Bergmann T., Fischer T. C., Schmitt H. R., Kraemer S. B., 2017, MNRAS, 469, 3286

Dors O. L., Jr., Storchi-Bergmann T., Riffel R. A., Schimdt A. A., 2008, A\&A, 482, 59

Eisenhauer F. et al., 2003, in Iye M., Moorwood A. F. M., eds, Proc. SPIE Conf. Ser. Vol. 4841, Instrument Design and Performance for Optical/Infrared Ground-based Telescopes. SPIE, Bellingham, p. 1548

Ferrarese L., Merritt D., 2000, ApJ, 539, L9

Ferrarese L., Livio M., Freedman W., Saha A., Stetson P. B., Ford H. C., Hill R. J., Madore B. F., 1996, ApJ, 468, L95

Filippenko A. V., 2003, 290, 369

Filippenko A. V., Sargent W. L. W., 1986, in Giuricin G., Mezzetti M., Ramella M., Mardirossian F., eds, Astrophysics and Space Science Library Vol. 121, Structure and Evolution of Active Galactic Nuclei. Springer-Verlag, Berlin, p. 21

Fluks M. A., Plez B., The P. S., de Winter D., Westerlund B. E., Steenman H. C., 1994, A\&AS, 105

Gebhardt K. et al., 2000, ApJ, 539, L13

Girardi L., Bressan A., Bertelli G., Chiosi C., 2000, A\&AS, 141, 371

González Delgado R. M., Heckman T., Leitherer C., 2001, ApJ, 546, 845

Gu Q., Melnick J., Cid Fernandes R., Kunth D., Terlevich E., Terlevich R., 2006, MNRAS, 366, 480

Heckman T. M., González-Delgado R., Leitherer C., Meurer G. R., Krolik J., Wilson A. S., Koratkar A., Kinney A., 1997, ApJ, 482, 114

Höfner S., Loidl R., Aringer B., Jørgensen U. G., Hron J., 2000, ESA SP-456, ISO Beyond the Peaks: The 2nd ISO Workshop on Analytical Spectroscopy, Villafranca del Castillo, Spain, p. 299

Ho L. C., Filippenko A. V., Sargent W. L. W., Peng C. Y., 1997, ApJS, 112, 391

Hsieh B. C. et al., 2017, ApJ, 851, L24
Jiménez-Bailón E., Santos-Lleó M., Mas-Hesse J. M., Guainazzi M., Colina L., Cerviño M., González Delgado R. M., 2003, ApJ, 593, 127

Kennicutt R. C., Jr., 1988, ApJ, 334, 144

Kennicutt R. C., Jr., Keel W. C., Blaha C. A., 1989, AJ, 97, 1022

Kormendy J., Ho L. C., 2013, ARA\&A, 51, 511

Kotilainen J. K., Forbes D. A., Moorwood A. F. M., van der Werf P. P., Ward M. J., 1996, A\&A, 313, 771

Krabbe A., Sternberg A., Genzel R., 1994, ApJ, 425, 72

Lançon A., Mouhcine M., 2002, A\&A, 393, 167

Lançon A., Wood P. R., 2000, A\&AS, 146, 217

Lejeune T., Cuisinier F., Buser R., 1997, A\&AS, 125

Lejeune T., Cuisinier F., Buser R., 1998, A\&AS, 130, 65

Ma C., de Grijs R., Ho L. C., 2018, ApJ, 857, 116

Maraston C., 2005, MNRAS, 362, 799 (M05)

Maraston C., Strömbäck G., 2011, MNRAS, 418, 2785 (M11)

Martins L. P., Riffel R., Rodríguez-Ardila A., Gruenwald R., de Souza R., 2010, MNRAS, 406, 2185

Martins L. P., Rodríguez-Ardila A., Diniz S., Gruenwald R., de Souza R., 2013a, MNRAS, 431, 1823

Martins L. P., Rodríguez-Ardila A., Diniz S., Riffel R., de Souza R., 2013b, MNRAS, 435, 2861

Mateus A., Sodré L., Cid Fernandes R., Stasińska G., Schoenell W., Gomes J. M., 2006, MNRAS, 370, 721

Mazzalay X. et al., 2013, MNRAS, 428, 2389

Mazzuca L. M., Knapen J. H., Veilleux S., Regan M. W., 2008, ApJS, 174, 337

Meneses-Goytia S., Peletier R. F., Trager S. C., Vazdekis A., 2015, A\&A, 582, A97

Menezes R. B., Steiner J. E., Ricci T. V., 2014, MNRAS, 438, 2597

Menezes R. B., da Silva P., Ricci T. V., Steiner J. E., May D., Borges B. W., 2015, MNRAS, 450, 369

Mollá M., Hardy E., Beauchamp D., 1999, ApJ, 513, 695

Origlia L., Oliva E., 2000, New Astron. Rev., 44, 257

Pickles A. J., 1998, PASP, 110, 863

Ramos Almeida C., Pérez García A. M., Acosta-Pulido J. A., 2009, ApJ, 694, 1379

Riffel R., Pastoriza M. G., Rodríguez-Ardila A., Maraston C., 2007, ApJ, 659, L103

Riffel R., Pastoriza M. G., Rodríguez-Ardila A., Bonatto C., 2009b, MNRAS, 400, 273

Riffel R., Ruschel-Dutra D., Pastoriza M. G., Rodríguez-Ardila A., Santos J. F. C., Jr, Bonatto C. J., Ducati J. R., 2011a, MNRAS, 410, 2714

Riffel R., Bonatto C., Cid Fernandes R., Pastoriza M. G., Balbinot E., 2011b, MNRAS, 411, 1897

Riffel R., Riffel R. A., Ferrari F., Storchi-Bergmann T., 2011c, MNRAS, 416, 493

Riffel R. et al., 2015, MNRAS, 450, 3069

Riffel R. A., 2013, Bol. Asoci. Argentina Astron. Plata Argentina, 56, 13

Riffel R. A., Storchi-Bergmann T., Dors O. L., Winge C., 2009a, MNRAS, 393, 783

Riffel R. A., Storchi-Bergmann T., McGregor P. J., 2009c, ApJ, 698, 1767

Riffel R. A., Storchi-Bergmann T., Riffel R., Pastoriza M. G., 2010, ApJ, 713, 469

Riffel R. A. et al., 2016, MNRAS, 461, 4192 (R16)

Röck B., Vazdekis A., Ricciardelli E., Peletier R. F., Knapen J. H., FalcónBarroso J., 2016, A\&A, 589, A73

Rodríguez-Ardila A., Mazzalay X., 2006, MNRAS, 367, L57

Rodríguez-Ardila A., Contini M., Viegas S. M., 2005, MNRAS, 357, 220

Rosario D. J. et al., 2018, MNRAS, 473, 5658

Rosenberg M. J. F., van der Werf P. P., Israel F. P., 2012, A\&A, 540, A116

Ryder S. D., Knapen J. H., Takamiya M., 2001, MNRAS, 323, 663

Schaller G., Schaerer D., Meynet G., Maeder A., 1992, A\&AS, 96, 269

Schinnerer E., Maciejewski W., Scoville N., Moustakas L. A., 2002, ApJ, 575,826

Schmidt A. A., Copetti M. V. F., Alloin D., Jablonka P., 1991, MNRAS, 249, 766

Schönell Astor J. J., Storchi-Bergmann T., Riffel R. A., Riffel R., 2017, MNRAS, 464, 1771 
Seo W.-Y., Kim W.-T., 2013, ApJ, 769, 100

Shlosman I., Frank J., Begelman M. C., 1989, Nature, 338, 45

Shlosman I., Begelman M. C., Frank J., 1990, Nature, 345, 679

Singh R. et al., 2013, A\&A, 558, A43

Storchi-Bergmann T., Raimann D., Bica E. L. D., Fraquelli H. A., 2000, ApJ, 544, 747

Storchi-Bergmann T., González Delgado R. M., Schmitt H. R., Cid Fernandes R., Heckman T., 2001, ApJ, 559, 147

Storchi-Bergmann T., Riffel R. A., Riffel R., Diniz M. R., Borges Vale T., McGregor P. J., 2012, ApJ, 755, 87
Valencia-S. M., Zuther J., Eckart A., García-Marín M., Iserlohe C., Wright G., 2012, A\&A, 544, A129

Westera P., Lejeune T., Buser R., Cuisinier F., Bruzual G., 2002, A\&A, 381, 524

Worthey G., Ottaviani D. L., 1997, ApJS, 111, 377

Zibetti S., Gallazzi A., Charlot S., Pierini D., Pasquali A., 2013, MNRAS, 428,1479

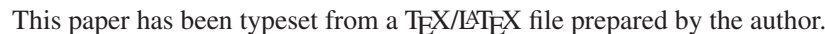

